\title{
Flight performance of EXAM - a balloon-borne detector to search for extragalactic antimatter
}

\author{
S.P. Ahlen ${ }^{a}{ }^{*}$, D. Loomba ${ }^{a}$, A. Marin ${ }^{a}$, B. Zhou ${ }^{a}$, Y.D. He ${ }^{b}$, P.B. Price ${ }^{b}$, \\ D.P. Snowden-Ifft ${ }^{b}$, C. Bower ${ }^{c}$, J.A. Musser ${ }^{\text {c }}$, T.E. Coan ${ }^{\mathrm{d}}$, G. Tarlé ${ }^{\mathrm{e}}$, A. Tomasch ${ }^{\mathrm{e}}$, \\ M.H. Salamon ${ }^{\text {f }}$, J.J. Beatty ${ }^{\mathrm{g}}$, D.J. Crary ${ }^{\mathrm{g}}$ \\ ${ }^{a}$ Physics Department, Boston Unversity, Boston, MA 02215, USA \\ 'Phystcs Department, University of California, Berkeley, CA 94720, USA \\ 'Physics Department, Indiana Unicersity, Bloomington, IN 47405, USA \\ ${ }^{d}$ Los Alamos National Laboratory, Los Alamos, NM 87545, USA \\ 'Phystcs Department, University of Michigan, Ann Arbor, MI 48109, USA \\ 'Physics Department, University of Utah, Salt Lake City, UT 84112, USA \\ ${ }^{g}$ Physics Department, Washington Unu'ersity, St. Louis, MO 63130, USA
}

(Received 5 October 1993; revised form received 13 December 1993)

We describe the performance of the EXAM detector during its five hour balloon flight in 1988. EXAM is an experiment designed to search for cosmic rays of extragalactic origin which are made of antimatter. The EXAM technique to identify antinuclei is unique, being based on higher order corrections to electronic stopping power of charged particles, and on the response characteristics of CR-39 track-etch detectors, plastic scintillators, and Cherenkov radiators. Included in the present paper are the completed analysis of the electronic detectors, and preliminary results of the analysis of the track-etch detectors, including a demonstration of our ability to match particles identified with the drift tube tracking elements during the flight with their tracks found in the passive CR-39 detectors. When the CR-39 analysis is complete, we will have approximately 10000 events for which antimatter analysis can be made.

\section{Introduction}

Ten years ago we developed the EXAM technique (for extragalactic antimatter) which was claimed to be capable in principle of directly identifying heavy antinuclei in the cosmic radiation [1]. The proposed technique was unusual in that it did not require a magnet or calorimeter, but relied on details of energy loss processes of relativistic charged particles in matter and the response characteristics of various types of particle detectors. The promise of the technique was that it would be capable of extending searches for antimatter in the cosmic rays to levels $\approx 10^{-6}$, lower than possible with calorimeters, magnets or geomagnetic techniques $\left(\approx 10^{-4}\right)$, even allowing for the fact that the EXAM technique is not sensitive to antinuclei with atomic number less than ten. This improvement by two orders of magnitude would be crucial to surpass the level of $\approx 10^{-5}$ expected for the fraction of extragalactic cosmic rays near the Earth [2]. For various theoreti-

* Corresponding author. cal and indirect experimental reasons most cosmologists reject the possibility of the existence of large quantities of antimatter outside of our galaxy. However, the fact remains that no experiment has been done which directly excludes extragalactic objects from being made of antimatter [2]. Furthermore, there is still no compelling model of how an initial matter-antimatter symmetric universe could have evolved to an anti symmetric one (baryo-genesis). The missing ingredients are evidence for baryon non-conserving processes, and a clear understanding of CP violation.

Subsequent to our initial proposal for EXAM we were funded to build the experiment. On 4 September 1988, at 8:00 AM local time, the experiment was launched from Prince Albert, Saskatchewan. It achieved an altitude of $36.0 \mathrm{~km}\left(5 \mathrm{~g} / \mathrm{cm}^{2}\right.$ residual atmosphere) at 11:00 AM. It remained at float altitude until 4:00 PM, at which time the flight was terminated to prevent the balloon from being swept by strong westerly winds into a wild and remote area for which recovery would have been difficult if not impossible. As such the flight was terminated well short of the $20 \mathrm{~h}$ requested, and of the time anticipated in the origınal 
proposal (which claimed an antimatter limit approaching $10^{-6}$ for several $40 \mathrm{~h}$ flights). Furthermore, about half the short flight was devoted to acquiring particles with small charge for calibration purposes and to tuning high voltages, so the time available for collecting the iron group nuclei which are most sensitive to the higher order corrections required for the antimatter search was only about $2.5 \mathrm{~h}$. Nevertheless we acquired sufficient data for a realistic evaluation of the technique, even though we do not have enough data to be able to surpass the current world record of $1.4 \times 10^{-4}$ for the limit on antinuclei with atomic number greater than nine [3].

\section{The EXAM instrument}

\subsection{Track-etch detectors}

Fig. 1 shows the left side and end views of the EXAM instrument as flown. The top detector consisted of 180 stacks of the track-etch detector known as CR-39 (including the additive DOP [4]). The stacks were arranged in a $9 \times 20$ pattern. Each stack held six

left side view

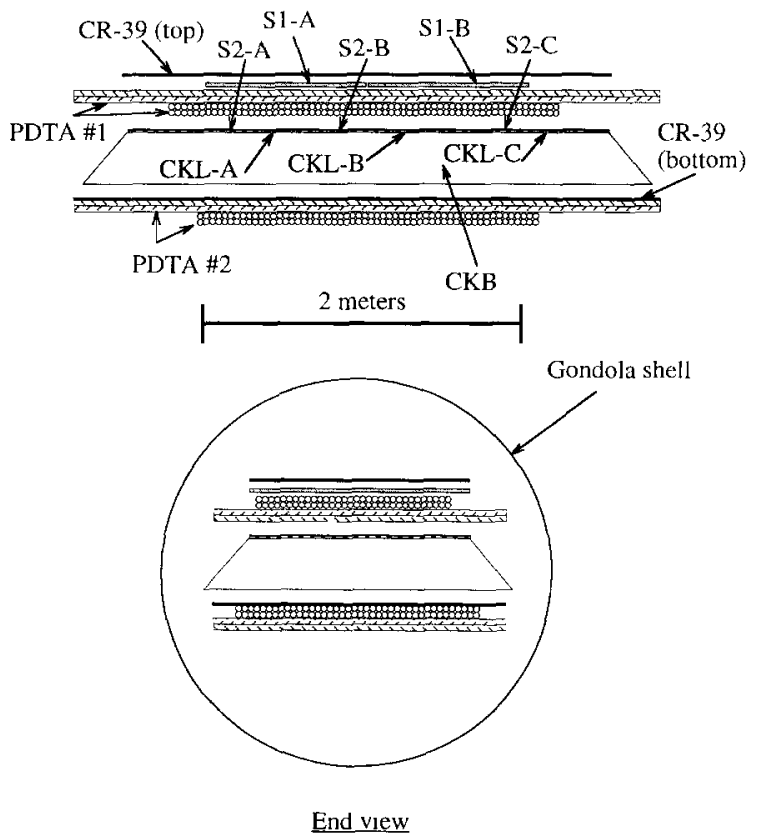

Fig. 1. Schematic of the side and end views of EXAM. CR-39 are track-etch detectors, $S 1$ and $S 2$ are scintillators, $C K L$ are slabs of Cherenkov radiators, CKB is the Cherenkov light integration box, and PDTA are proportional drift tube arrays. sheets of CR-39 with dimensions $15.24 \times 15.24 \times 0.0254$ $\mathrm{cm}^{3}$ for a total of 1080 sheets. The total area covered was $4.18 \mathrm{~m}^{2}$. The stacks were mounted on a $1.05 \mathrm{~cm}$ thick aluminum honeycomb panel.

Below the Cherenkov light integration box (described below) was the second layer of CR-39 stacks. The mounting and construction was similar to that of the top CR-39 array. The total number of stacks that could be loaded on this layer was 288 , in a $12 \times 24$ pattern. However, only 192 stacks were loaded (in a $12 \times 16$ pattern) due to a reduction of the experiment sensitive area shortly before the flight. This reduction was necessitated by a $200 \mathrm{~kg}$ weight reduction imposed by the balloon launching organization several months prior to the flight. As with the top CR-39 layer, six sheets were placed in each stack, for a total of 1152 sheets. The area covered by the bottom CR-39 was $4.46 \mathrm{~m}^{2}$.

\subsection{Scintillators}

Under the top CR-39 array was S1, consisting of two adjacent slabs, S1-A and S1-B, of $2.54 \mathrm{~cm}$ thick Bicron BC-412 scintillator. Originally there were three such slabs, but one was removed due to the weight reduction requirement mentioned above. Each slab had dimensions $100.1 \times 137.9 \mathrm{~cm}^{2}$. The left and right sides of each detector segment were coupled via adiabatic light pipes to six RCA 4900 photomultiplier tubes (PMTs), which had $7.62 \mathrm{~cm}$ diameter photocathodes, and which were enclosed in magnetic shields. To prevent space-charge saturation for the heavily ionizing flight events, the PMT divider chain utilized only the first six dynodes of the tube. The two slabs were separately wrapped in electrostatic shielding material, which had a black opaque plastic exterior, and a dull metallic interior. Four charge sensitive preamplifiers were connected to each slab, with two groups of three PMTs on each side of each slab being passively summed at the preamp inputs. Each preamp was fed to a fast shaping amplifier. The two shaping amps on each side were fed to a linear fan-in, with two outputs, one going to a discriminator, and the other to a 10-bit peak sensing analog to digital converter (ADC). The discriminator was used in the event trigger logic and to provide a stop signal for a time to digital converter (TDC). Tests with sea level cosmic rays demonstrated a collection efficiency of about 1000 photoelectrons per singly charged minimum ionizing particle at normal incidence. Tests with ${ }^{241} \mathrm{Am} \alpha$ particles showed that the standard deviation of collection efficiency of the two sides summed together was $7 \%$ over the entire surface of an S1 slab.

The detector labeled $\mathrm{S} 2$ in Fig. 1 consisted of three slabs of BC-412 plastic scintillator, S2-A, S2-B, and S2-C, each $0.61 \mathrm{~cm}$ thick, and each with dimensions 
$100.1 \times 137.9 \mathrm{~cm}^{2}$. Each side of each slab was coupled to two magnetically shielded RCA 4900 PMTs via adiabatic light pipes. Divider chains for six dynodes, similar to those for S1, were used for the S2 tubes. The three slabs of $\mathbf{S} 2$ were separately wrapped with the same type of material as used for S1. For S2, the pair of PMTs on each side was fed to a single preamp and shaping amp. The shaping amp output went to a discriminator and a peak sensing ADC. The discriminator was used in the event trigger logic and to provide a stop signal for a TDC. Tests with sea level cosmic rays demonstrated a collection efficiency of about 300 photoelectrons per singly charged minimum ionizing particle at normal incidence. Tests with ${ }^{241} \mathrm{Am} \alpha$ particles showed that the standard deviation of collection efficiency of the two sides summed together was $11 \%$ over the entire surface of an S2 slab.

The event trigger was determined by the coincidence of any side of any S1 slab, AND any side of any S2 slab. This was a soft trigger, designed primarily for safety in the event of the failure of electronics on one side of a scintillator slab. It had the added benefit of enabling a large number of oxygen nuclei to trigger the instrument, providing a valuable source of calibration data. The PMT TDC data provided crude time-of-flight information (with a standard deviation of $1.9 \mathrm{~ns}$, after timing walk corrections).

\subsection{Cherenkov detector}

Directly below $\mathrm{S} 2$ was the Bicron BC-480 Cherenkov radiator, consisting of three slabs, CKL-A, CKL-B, and CKL-C. Each of the slabs was $1.27 \mathrm{~cm}$ thick, with dimensions $100.1 \times 137.9 \mathrm{~cm}^{2}$. Each side of each slab was coupled to four magnetically shielded RCA 4900 PMTs via adiabatic light pipes. The CKL PMTs utilized bases with divider chains providing anode outputs. One side of each of the slabs was covered by white nitrocellulose filter paper having a diffuse reflectivity of $95 \%$, which was backed by the same opaque material used to wrap the scintillators. The other sides of the slabs viewed the interior of the light collection box described below. The four PMTs on each side of each slab were passively summed at the input of a charge sensing amplifier, whose output went to a fast shaping amplifier. The outputs of the shaping amps went to ADC inputs.

The notation used for the 16 ADC channels associated with the left and right sides of each of the scintillator and Cherenkov slabs is illustrated in Fig. 2 (e.g. sla1 is the ADC signal for the sum of the six PMTs on the left side of S1-A, i.e. the one with the small value of the $y$ coordinate).

To reduce the dependence of light collection efficiency on energy and angle of incidence, a light integration box (LIB) was used to collect light escaping

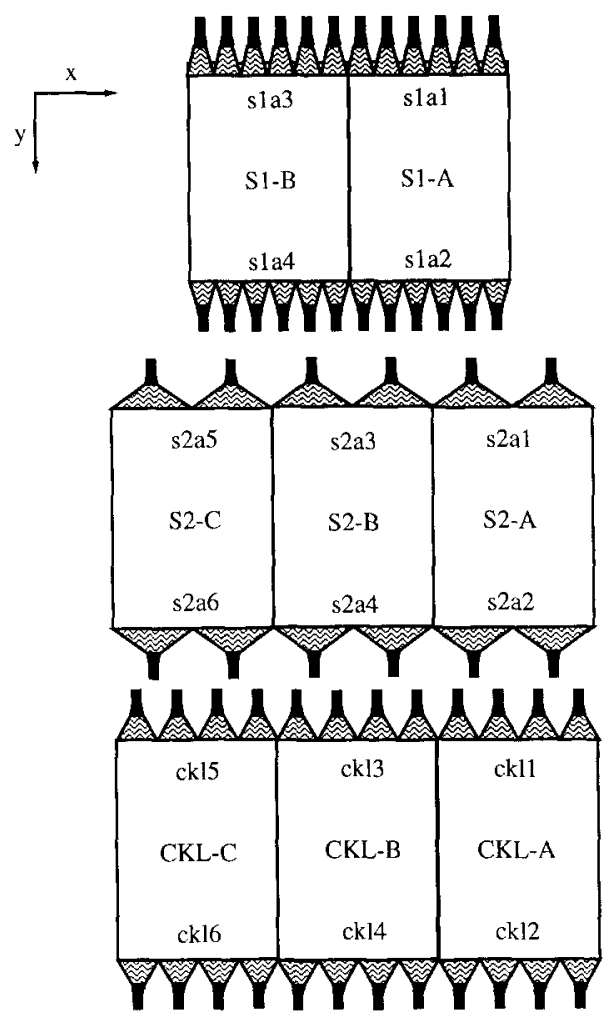

Fig. 2. Top view of the five scintillator slabs and three Cherenkov slabs, indicating notation used to characterize the 16 ADC channels for the light piped signals.

from the Cherenkov radiators. The LIB was a truncated pyramid open at the top, where the Cherenkov radiators were mounted side by side. It was $37.8 \mathrm{~cm}$ high, $135 \times 316 \mathrm{~cm}^{2}$ at the top, and $182 \times 359 \mathrm{~cm}^{2}$ at the base. The base plate was an aluminum honeycomb panel to minimize weight. A top view of the complete Cherenkov detector is shown in Fig. 3a. Magnetically shielded RCA S83006E PMTs with $11.1 \mathrm{~cm}$ diameter photocathodes were mounted to the side of the LIB through the use of flanges and O-ring light tight seals. These PMTs used the same divider chain as the tubes viewing the CKL slabs directly. The LIB had space for 40 PMTs, but only 20 were used for the flight to reduce weight. Groups of three or four PMTs were passively summed at the inputs to a total of six charge sensing preamplifiers. The six preamp outputs were fanned in to summing amplifiers, with a single output that was fed to a shaping amplifier. The fractional area of the LIB covered by 40 PMTs was $3.3 \%$, and the slant angle of $40^{\circ}$ prevented these PMTs from viewing the CKL slabs directly. The sides of the LIB were painted with $\mathrm{BaSO}_{4}$ paint [5] having a reflectivity of $97 \%$. For coverage with 40 (20) PMTs the collection efficiency of 

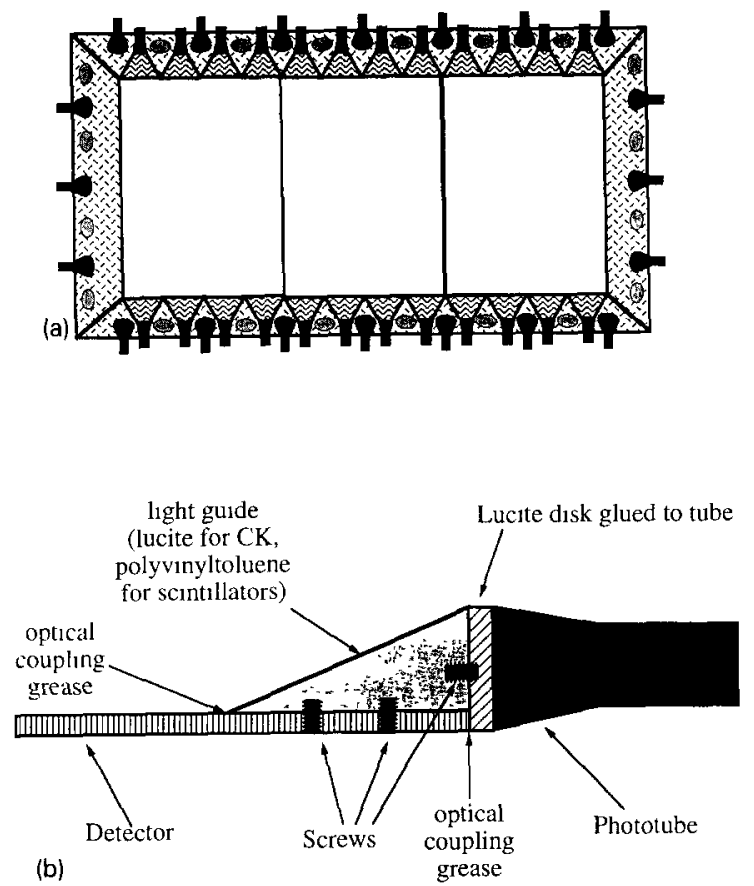

Fig. 3. (a) Top view of Cherenkov detector. For the flight, 20 PMTs viewed the interior of the light integration box, and 24 PMTs viewed the radiator slabs via adiabatic light pipes. The shaded ellipses show where additional PMTs would be mounted if the weight saving measures for the flight were not required. (b) Technique for attaching PMTs to light pipes on CKL, S1. and S2 slabs.

the LIB was $40 \%(25 \%)$. Tests with sea level cosmic rays have shown that with 40 PMTs on the LIB, 17 photoelectrons were collected from the LIB, and 17 from the light piped PMTs for relativistic singly charged particles. These tests also showed that the total summed Cherenkov signal was independent of position and angle of incidence at the several percent level. Flight data showed that the cross-talk between different CKL slabs was quite small: that is, when one slab was hit almost all the light was collected by the light pipes on that slab and the LIB, with no more than a few percent appearing at the light pipes of other slabs.

\subsection{Light piping technique}

Fig. 3b illustrates the novel approach we took in the mounting of the PMTs to the edges of the scintillators and Cherenkov radiators. To maintain strength without recourse to external mounting brackets, we chose to use the detectors themselves as parts of the light pipes. To these were attached by means of screws and inserts the main parts of the light pipes. Optical coupling grease was spread in the region between the light pipe and detector. To attach the PMTs, we glued disks of polymethylmethacrylate to the tubes, mounted inserts and screws into the disks, and screwed the PMTs into inserts in the end of the light pipes [6]. This provided a robust mechanical mounting system which had the added benefit of making PMT replacement a trivial procedure. Optical grease was placed on the surface between the disk and the light pipe. The size of the screws is exaggerated in Fig. 3b. The reduction in light collection due to the obscuring screws was of the order of $1 \%$.

\subsection{Drift tube system}

The drift tube system provided active tracking data to be used to match events identified with the electronic detectors with those in the CR-39 detectors. Originally, the instrument contained 416 tubes, but 32 were removed for the weight reduction reasons referred to above. The tubes were of two types: long and short. The short tubes were $182 \mathrm{~cm}$ long, and the long tubes were $368 \mathrm{~cm}$ long. Each type had round stainless steel walls with an inner diameter of $3.81 \mathrm{~cm}$, and a wall thickness of $0.0154 \mathrm{~cm}$. Tungsten wires plated with gold (6\% by weight) with $51 \mu \mathrm{m}$ diameter were strung without bridges the length of the tubes. The tubes were built in modules of 16 tubes each ( 8 on top and 8 directly below them). The tubes and wires were fixed at each end of an aluminum end-block by a set of concentric plugs and pins which maintained the positional accuracy of a wire center with respect to that of any other in the same module to about $40 \mu \mathrm{m}$. The wires were mounted by both crimping and soldering. The end-blocks had provisions for mounting to the detector frame so that the drift tubes were located with a tolerance of about $2 \mathrm{~mm}$ relative to all other elements of the experiment. They were built with internal gas passages to provide a series gas flow system for each module of 16 tubes. The gas plumbing was configured to provide two independent series systems for the 384 tubes. The flight gas was a mixture consisting of argon $(90 \%), \mathrm{CO}_{2}(9 \%)$ and $\mathrm{CH}_{4}(1 \%)$. Its pressure was maintained about $0.01 \mathrm{psi}$ above that of the gondola interior during the flight through the use of mineral oil bubblers.

Care was taken to apply sufficient tension to the wires to maintain deflection at the center of the wire to less than $250 \mu \mathrm{m}$ (including the electrostatic effects of the high voltage applied to the wire). This was checked after all modules were built by an electro-mechanical technique based on resonance of the wire induced in a magnetic field by an alternating current [7]. The small number of drift tubes failing the initial check were re-strung and re-tested before being mounted in the instrument. 


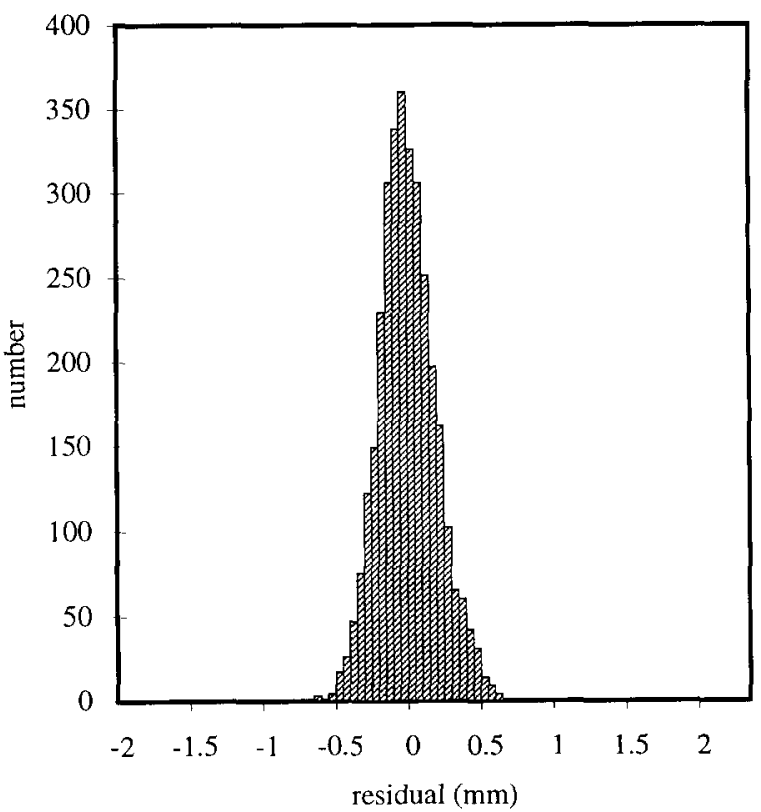

Fig. 4. Residuals of drift tubes determined with sea level muon tests. Single tube standard deviation is $175 \mu \mathrm{m}$.

Amplifier-comparator cards were built and mounted on the ends of the drift tube modules. The comparator threshold was set to $1 / 3$ of the peak signal of a relativistic iron nucleus. This setting was made to achieve a balance between too low a discriminator setting, which would result in excessive numbers of spurious triggers on delta rays for the heavily charged particles like iron [8], and too high a setting, which would result in reduced efficiency for lowly charged particles like neon, which are near the sensitivity limit of the antimatter detection scheme.

The drift tube TDC system was used in a common stop mode, with 9-bit resolution, and with a sensitivity of $3.25 \mathrm{~ns}$ per channel. The maximum drift time for flight conditions was calculated to be $0.88 \mu \mathrm{s}$, about half the range for the TDC configuration.

The drift tube modules were used to form two arrays, with a mean vertical separation of $68.9 \mathrm{~cm}$. Each array was composed of a set of two rows of short tubes oriented at right angles to two rows of long tubes. The top array consisted of 64 long tubes and 128 short tubes. The bottom array had 80 long tubes and 112 short tubes (the 32 tubes which were removed to save weight were single 16 tube modules located at either end of the lower short tube array). To remove left-right tracking ambiguities the wire centers of four rows of parallel tubes of the top and bottom arrays did not lie along the same vertical lines.
Fig. 4 illustrates the inherent resolution capabilities of the drift tubes, determined with sea level cosmic rays for one short module oriented on its side in a bench test, so that tracks with eight tubes could be obtained. The data imply a single tube standard deviation of $175 \mu \mathrm{m}$.

\subsection{Data acquisition}

An on-board LSI 11/23 microprocessor controlled data acquisition, and sent data asynchronously in sequences of 50 words over the CAMAC dataway to a custom built pulse code modulation (PCM) encoder which resided in the main CAMAC crate (a second CAMAC crate was devoted to the drift tube TDC system). The 50 word sequences were prefixed at the PCM encoder with a user-defined synchronization word and a blank word, and then sent in bi-phase to two on-board transmitters, from which they were sent to Earth at $64 \mathrm{kbit} / \mathrm{s}$, where they were received by the ground support station. This consisted of a PCM decoder, a PDP 11/73 computer, and a Cypher 3200 bpi tape drive. During flight, the ground support system was used to monitor the performance of the detectors, which allowed us to make appropriate modifications to PMT and drift tube high voltage with a set of up-link commands. Four types of data frames were telemetered to the ground: 1) a primary data frame consisting of ADC and TDC values, and identification codes for the hit detectors; 2 ) an overflow frame to contain event data which would not fit in the primary frame (in case large numbers of drift tubes and/or PMT channels were hit);3) a housekeeping data frame to read out data on temperatures, pressures, flow rates, drift tube currents, low voltages and high voltages; 4) calibration data frames to monitor performance of the TDC and ADC systems.

\subsection{Power system}

Lithium batteries were used with large capacity voltage regulators to provide instrument power for the NIM and CAMAC crates, amplifiers, and high voltage supplies. The total power dissipated was $870 \mathrm{~W}$.

\subsection{Gondola characteristics}

The instrument frame was designed to slide along rails from an external support cart onto rails inside of the elongated EXAM gondola. Preparation of the instrument was done on the external cart, which was surrounded by a large black tent to minimize light leaks. The gondola shells consisted of three parts: two hemispherical spun aluminum end-caps and an aluminum cylindrical mid-section. All wall thicknesses were about $2 \mathrm{~mm}$. The diameter of all three elements 
Table 1

Weights of components of EXAM

\begin{tabular}{lc}
\hline Item & Weight [kg] \\
\hline Drift tubes, CK, S1, S2, PMTs, frame & 1120 \\
CR-39 with honeycomb support & 60 \\
Two CAMAC and two NIM bins & 90 \\
Power and control systems & 40 \\
Gas bottle and regulator & 20 \\
Drift tube plumbing & 20 \\
Glass track-etch detectors & 20 \\
Two gondola end caps & 160 \\
Gondola mid-section & 370 \\
Spreader bar & 50 \\
Rigging & 40 \\
Parachute & 205 \\
CIP and batteries & 127 \\
Terminate box & 13 \\
Crush pad & 13 \\
Valves for balloon & 11 \\
Ballast & 141 \\
Total & 2500
\end{tabular}

was $244 \mathrm{~cm}$. The length of the assembled shell was 720 $\mathrm{cm}$. The entire surface of the gondola was covered with $2.54 \mathrm{~cm}$ of white Ethafoam. The experiment's internal gaseous environment consisted of normal air at slightly elevated pressure (so we could check for leaks). Exhaust gas from the drift tube system was dumped to the interior of the gondola. The gondola volume was so large $\left(28 \mathrm{~m}^{3}\right)$ that no attempt was made to purge the interior of oxygen or water vapor. It was believed that the instrument would be warm enough so that condensation would not be a hazard. And the drift tube gas mixture that was used was chosen for safety reasons to eliminate the possibility of an explosion.

The weights of the various elements of EXAM are listed in Table 1.

\subsection{Geometrical factor}

As flown, the geometrical factor of EXAM was 4.2 $\mathrm{m}^{2}$ sr. Had the various elements not been removed, the geometrical factor would have been $5.7 \mathrm{~m}^{2} \mathrm{sr}$.

\section{Description of flight}

Several details of the flight have already been given in section 1. During the flight, approximately 600000 events were acquired. Fig. 5 shows environmental data for the air temperature and pressure of the interior of the gondola shells as functions of event number. The steadily increasing temperature reflects the fact that the launch occurred in the morning. The decline in

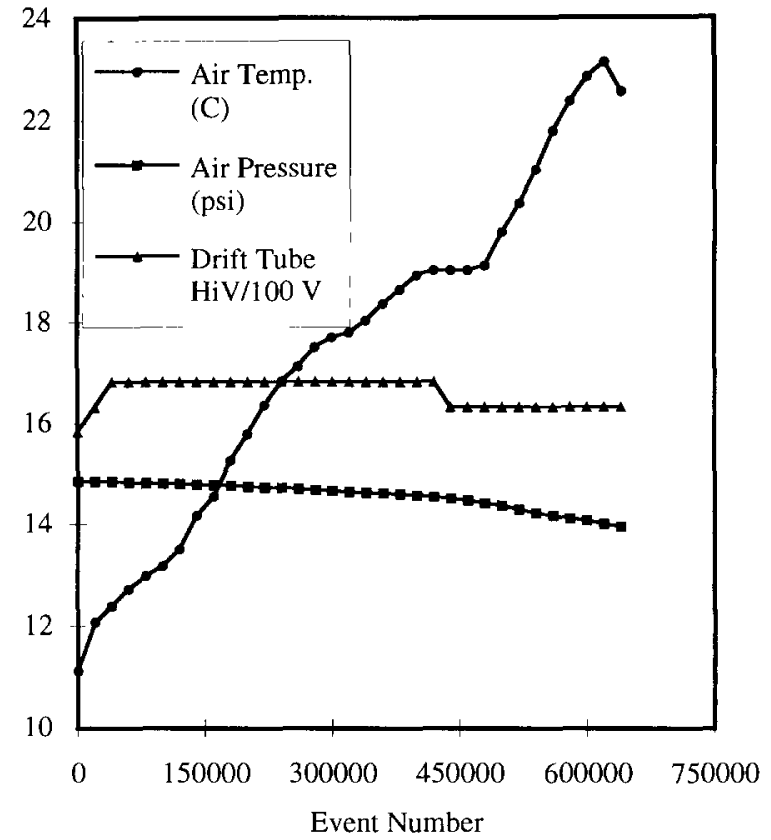

Fig. 5. Variations in temperature, pressure, and drift tube high voltage during flight of EXAM.

pressure reveals the presence of a small leak at the gondola shells. Also shown are data on the high voltage setting for the drift tubes, which were increased in the beginning of the flight to improve efficiency of the drift tubes for the lighter species. The drift tube volt-

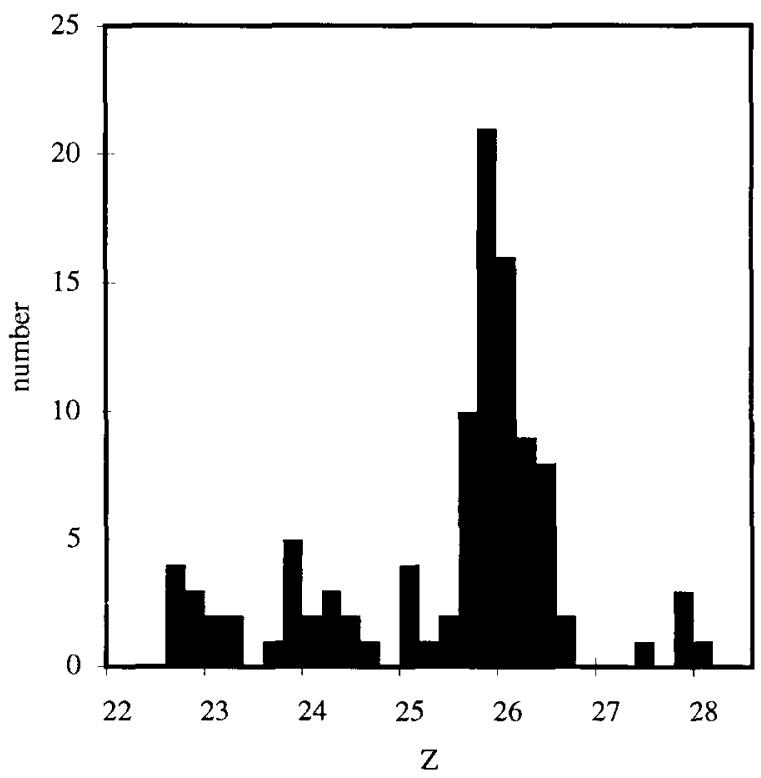

Fig. 6. CR-39 resolution from 1980 balloon flight. 
Table 2

Periods during EXAM flight with ten different high voltage combinations on $\mathrm{S} 1$ and $\mathrm{S} 2$

\begin{tabular}{lcll}
\hline $\begin{array}{l}\text { Time } \\
\text { Period }\end{array}$ & Event numbers & $\begin{array}{l}\text { S1 High } \\
\text { Voltage }\end{array}$ & $\begin{array}{l}\text { S2 High } \\
\text { Voltage }\end{array}$ \\
\hline I & 0 to 57612 & 830 & 1164 \\
II & 69683 to 86445 & 801 & 1164 \\
III & 90677 to 204150 & 773 & 1164 \\
IV & 213370 to 230210 & 773 & 1119 \\
V & 237100 to 269850 & 773 & 1069 \\
VI & 278870 to 332550 & 773 & 971 \\
VII & 341400 to 344850 & 750 & 971 \\
VIII & 361250 to 385120 & 750 & 1020 \\
IX & 394610 to 435000 & 702 & 1020 \\
X & 440770 to 635000 & 652 & 1020 \\
\hline
\end{tabular}

age was decreased later in the flight to compensate for the reduced gas density, due to the gondola leak, thus maintaining a nearly constant ratio of electric field to density (and thus minimizing variations in drift velocity throughout the flight).

The temperature variation of $12^{\circ} \mathrm{C}$ throughout the flight is small enough so that corrections can be applied to track etch-rate to compensate for the temperature dependence of track etching in the CR-39. Temperature sensors were placed at 12 evenly spaced intervals on each of the CR-39 frames to permit good knowledge of the temperature at the time of exposure. The observed variation is small enough so as not to have a serious effect even if not corrected for. As an illustration of this, Fig. 6 shows data from a 1980 balloon flight carried out by three of us (Ahlen, Price, and Tarlé). For this flight, several sheets of CR-39 were placed above a $1 \mathrm{~cm}$ thick slab of lead, and several sheets were placed below the lead. By comparing the cone size in the three surfaces above the lead with those in two surfaces below the lead, charge and velocity were determined. The expected charge resolution of $0.12 \mathrm{e}$ was degraded to $0.25 \mathrm{e}$ due to the $-8^{\circ} \mathrm{C}$ to $16^{\circ} \mathrm{C}$ temperature variations during the flight. Since the EXAM flight had half the temperature variation of this flight, one expects the EXAM charge resolution to be no worse than $0.2 \mathrm{e}$ even if temperature is not corrected for.

The CKL and CK-LIB high voltages were fixed throughout the flight at $1159 \pm 2 \mathrm{~V}$. The S1 and S2 high voltages were varied to optimize the early and late flight stages for acquisition of low charge calibration events and iron group events respectively. Table 2 lists the various stages of the flight and the corresponding high voltage settings for S1 and S2.

Periods I to VIII and periods IX to $\mathrm{X}$ each had time durations of approximately $2.5 \mathrm{~h}$.

\section{Analysis of electronic detectors with flight data}

Preliminary analyses of the electronics detectors were done at Berkeley, Boston, and Michigan from 1989 to 1991 [9-11]. Preliminary analysis of the CR-39 detectors was carried out at Berkeley and Michigan during the same period $[9,11]$. Below we present a complete analysis of the electronic detectors carried out at Boston in 1993.

The initial phase of the analysis required the development of an efficient drift tube tracking program. This program filtered out bad drift tubes by requiring any hit tube center to be closer than $5.08 \mathrm{~cm}$ and two standard deviations to the median position of the tubes that were hit in the pair of planes closest to the tube in question (including its own plane). If after this filter there were tubes hit in at least three of the four planes for the appropriate projected view, a track was fit to the tangents of circles whose radii were determined by the drift distance vs. drift time function (which was calculated using measured drift velocity data [12]). No more than one tube was used per plane, the tube used being selected arbitrarily if more than one tube survived the filter. In principle, the line representing the particle trajectory can go on either side of the circle corresponding to the impact parameter. Thus there are 16 possible combinations of hit points for four-plane
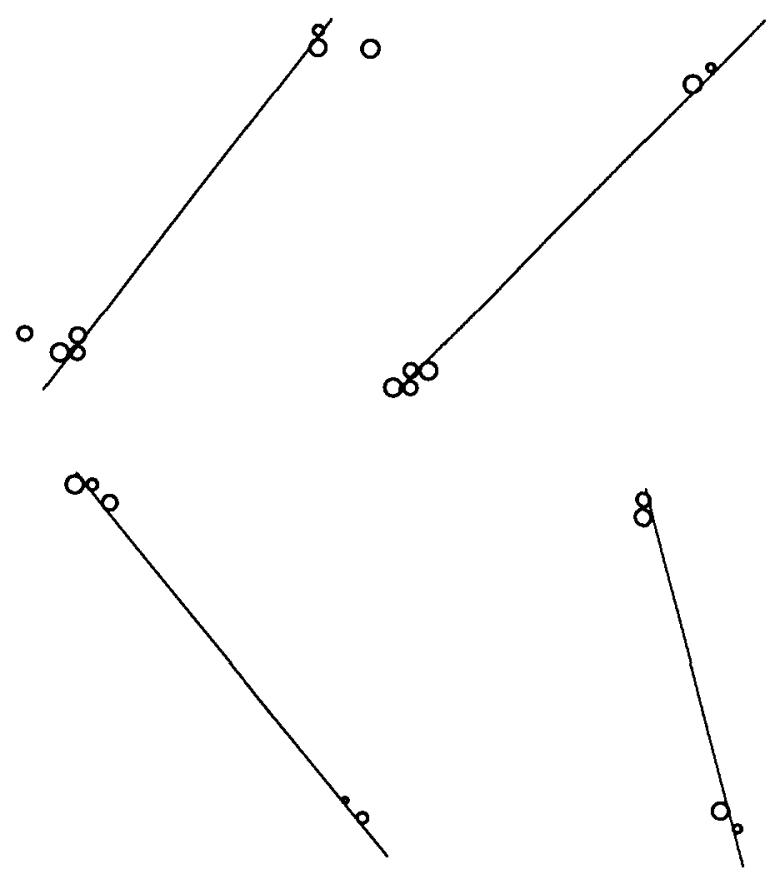

Fig. 7. Typical fits of drift tube data for silicon events. The circles have radii given by the drift time and the calculated time-to-space function. The lines are the fits that were calculated by the high efficiency tracking program. 
events, and 8 possibilities for three-plane events. The fitting was done by using the best least squares fit track of those determined for each of the 16 or 8 possibilities. Corrections for angle were made so that a simple $x$ or $y$ vs $z$ ( $z$ being the vertical coordinate) fitting procedure was appropriate (the angle was estimated by using the best of four fits using only the top and bottom tubes to determine possible trajectories, and the other two tubes or one tube to determine residuals). Fig. 7 shows typical drift tube track fits for fast silicon events. Since there was no need for resolution better than $1 \mathrm{~mm}$, no attempt was made to correct for PMT timing walk, scintillation light transit time, etc., since these effects were all small compared to the maximum drift time.

With the drift tube tracking information we were able to determine two types of geometric maps of scintillator response:

1) Maps were made in which the ratios of s1a1/s1a2, s1a3/s1a4, s2a1/s2a2, s2a3/s2a4, and $\mathrm{s} 2 \mathrm{a} 5 / \mathrm{s} 2 \mathrm{a} 6$ were determined as functions of position in the scintillators (the notation used to describe the sides of the scintillators, sla1 etc., is defined in Fig. 2). Prior to doing this, it was established that ADC and PMT response was linear, and event periods and detectors were identified in which performance was consistent. Data from stable periods and detectors were used to determine the ratio maps. Fig. 8 shows these maps.
2) Maps were made of the spatial dependence of the geometric mean of the signals from the two sides of the scintillator slabs. These were determined using fast oxygen data. Data from the stable periods and detectors for the entire flight were used (and were corrected for the variations in high voltage settings). Fig. 9 shows the geometric mean maps. They are normalized to those signals for fast oxygen at normal incidence for $830 \mathrm{~V}$ for S1 and $1164 \mathrm{~V}$ for S2. In what follows we will denote geometric mean by the abbreviation "gm". We will denote s1a1, s1a3, s2a1, s2a3, s2a5, ckl1, ckl3, and ckl5 as left sides of the detectors and the other sides as right sides. The geometric mean of S1-A is therefore defined, for example, as:

$\mathrm{S} 1 \mathrm{Agm}=\sqrt{\mathrm{s} 1 \mathrm{a} 1 \times \mathrm{s} 1 \mathrm{a} 2}$.

With the scintillator maps and drift tube tracking information we were able to complete the analysis of the electronic data. Cuts that were applied to the data are indicated in Table 3, along with the numbers of events remaining at successive stages. In Table 3 , note that $x$ planes refers to the tracking projection perpendicular to the short drift tubes (so the coordinates of the particle track are determined for this view by the short tubes). Similarly, $y$ plane refers to the long drift tubes.

In subsequent sections, we will use the following notation for sorted data according to scintillator slab

Table 3

Stages of analysis of electronic data

\begin{tabular}{|c|c|c|}
\hline Stage & Description & Events left \\
\hline Pass 0 & Data events off tape & 590798 \\
\hline Pass 1 & Events with $<16$ drift tubes & 589046 \\
\hline Pass 2 & Events with $>2 y$ planes and $>2 x$ planes hit & 180419 \\
\hline Pass 3 & Events cut if occurred during voltage changes on $\mathrm{S} 1, \mathrm{~S} 2$ & 158965 \\
\hline Pass 4 & Require both sides hit of $1 \mathrm{~S} 1$ slab and $1 \mathrm{~S} 2 \mathrm{slab}$ & 61677 \\
\hline Pass 5 & Concatenation of Pass 4 files & 61677 \\
\hline Pass 6 & Require hits on 2 of all S1 sides, and 2 of all S2 sides & 60785 \\
\hline Pass 7 & Require all $\mathrm{S} 1$ and $\mathrm{S} 2$ signals to be non-saturated at $\mathrm{ADC}$ & 50797 \\
\hline Pass 8 & Trajectories calculated from drift tube data of Pass 7 & 50797 \\
\hline Pass 9 & Eliminate events which fail filter & 40835 \\
\hline Pass 10 & Concatenation of Pass 9 files & 40835 \\
\hline Pass 11 & Sort events as $\mathrm{S} 1 \mathrm{~A} / \mathrm{S} 2 \mathrm{~A}, \mathrm{~S} 1 \mathrm{~A} / \mathrm{S} 2 \mathrm{~B}, \mathrm{~S} 1 \mathrm{~B} / \mathrm{S} 2 \mathrm{~B}$ or $\mathrm{S} 1 \mathrm{~B} / \mathrm{S} 2 \mathrm{C}$ & 40778 \\
\hline Pass 12 & Require drift tube track in correct S1 slab (check only $x$ ) & 36842 \\
\hline Pass 13 & Require drift tube track in correct $\mathrm{S} 1, \mathrm{~S} 2$ slabs (check $x, y$ ) & 35888 \\
\hline Pass 14 & Require $50.8 \mathrm{~cm}<x<254 \mathrm{~cm}$ at $\mathrm{S} 2$ & 35865 \\
\hline Pass 15 & Use scintillator maps to calculate S1L, S1R, S2L, S2R & 35865 \\
\hline Pass 16 & Sort data according to S1, S2 high voltages & 35865 \\
\hline Pass 17 & Require $\mathrm{SL}=\mathrm{SR}$ within $10 \%$ and $\mathrm{S} 1=\mathrm{S} 2$ within $10 \%$ & 22734 \\
\hline Pass 18 & Time periods I to VIII with CK-LIB > 100 channels & 11539 \\
\hline Pass 19 and 20 & Time periods IX and X with CK-LIB > 100 channels & 5419 \\
\hline Pass 21 & Pass 18 with bad CKL hits eliminated & 11439 \\
\hline Pass 22 and 23 & Pass 19 and 20 with bad CKL hits eliminated & 5368 \\
\hline Pass 21-23 & Data surviving all cuts & 16807 \\
\hline
\end{tabular}


combinations: 1) corresponds to the pair S1-A and S2-A; 2) corresponds to S1-A and S2-B; 3) corresponds to S1-B and S2-B; and 4) corresponds to S1-B and S2-C. Thus, for example, when we refer to Pass 13.3 data we mean those events after Pass 13 which corresponded to hits of the slabs S1-B and S2-B.

Note that the cuts which eliminated the most events were those on the number of drift tube planes hit and on the number of scintillator sides hit. This is due to the fact that the majority of flight triggers were due to carbon and oxygen nuclei which had poor efficiencies for triggering a large number of drift tubes and for making large enough scintillator signals to be above the discriminators for both sides of the detectors.

\subsection{Drift tube performance}

In Fig. 10, we show the distribution of total drift tubes hit per event from Pass 13.3 data (a perfect event would have eight tubes hit). The data are sub-divided into those events from the first half of the flight (roughly periods I to VIII) and those from the second half

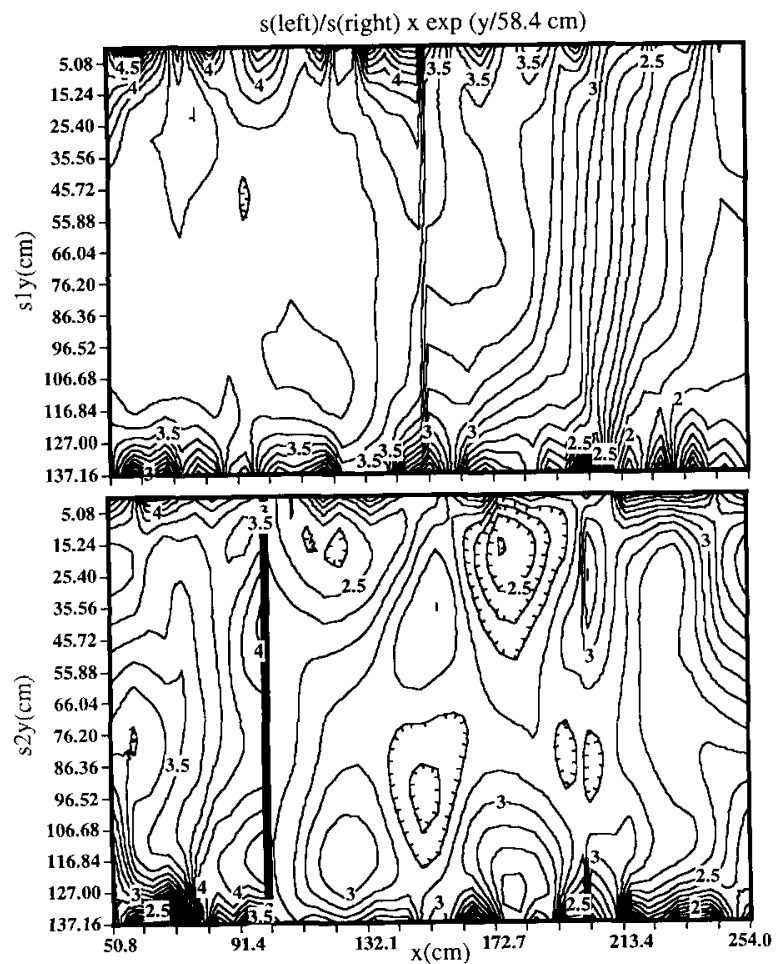

Fig. 8. Ratio maps for scintillators. Plotted are values of the ratio of the left to right scintillator signals for S1 and S2. The ratios are multiplied by a factor to eliminate the regular exponential attenuation behavior (the scintillators were found to have an attenuation length of about $117 \mathrm{~cm}$ ). For a perfectly uniform scintillator (except for the normal attenuation), the values plotted should all be equal to 3.23 .
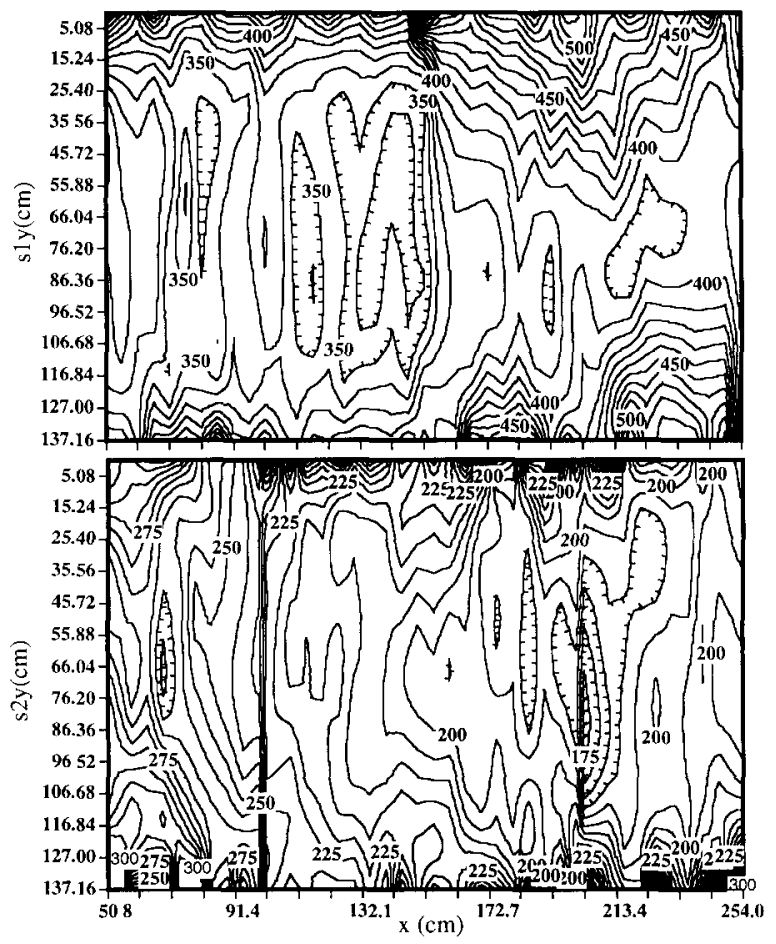

Fig. 9. Geometric mean map for scintillators. Plotted are values of the geometric mean for fast oxygen events in S1 and $\mathrm{S} 2$ at normal incidence, normalized to $830 \mathrm{~V}$ for S1 and 1164 $\mathrm{V}$ for $\mathrm{S} 2$. If normal attenuation were the only cause for spatial variation, the maps would be flat. Units are in ADC channel numbers.

(periods IX and $\mathrm{X}$ ). This is convenient since the first half of the flight consisted principally of particles in the neon to silicon region, while in the latter half of the flight, most particles were in the calcium to iron region. For this histogram, there does not seem to be any difference between the two classes of events.

Fig. 11 shows the relative frequency of hits for all the drift tubes through Pass 13. Note there were a few noisy tubes and a few tubes missing, including a single module denoted by tube numbers 161 to 169 and 225 to 232 (which probably had a failed power connection at the 16 channel amplifier-comparator card which plugged onto the module). The eight rows of tubes can be identified by the bumps in the histogram, which correspond to, with increasing tube numbers respectively, the top, second, third, and bottom rows of the long tubes, and the top, second, third, and bottom rows of short drift tubes. The gaps just before tube numbers 281 and 353 are due to the removal of the two drift tube modules that was mentioned above. In the track fitting, no attempt was made to exclude noisy tubes, as most of these were effectively removed by our filter. 


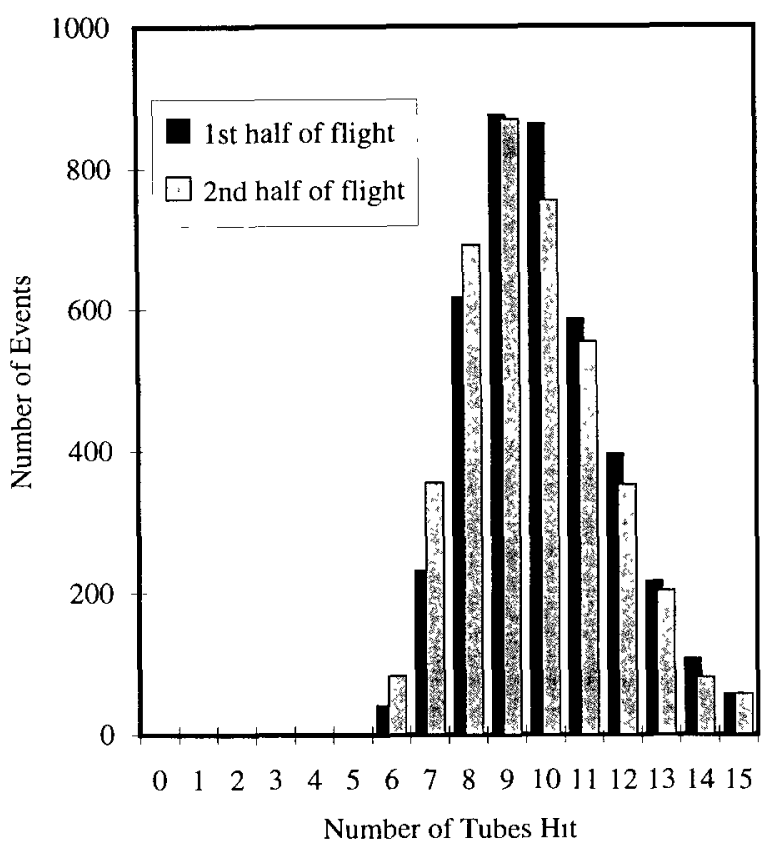

Fig. 10. Number of total drift tubes hit per event for first and second halves of flight (from Pass 13.3 data).

The quality of drift tube performance is illuminated by the histogram of TDC channel hits. Since the tubes were used in common stop mode, large TDC channels

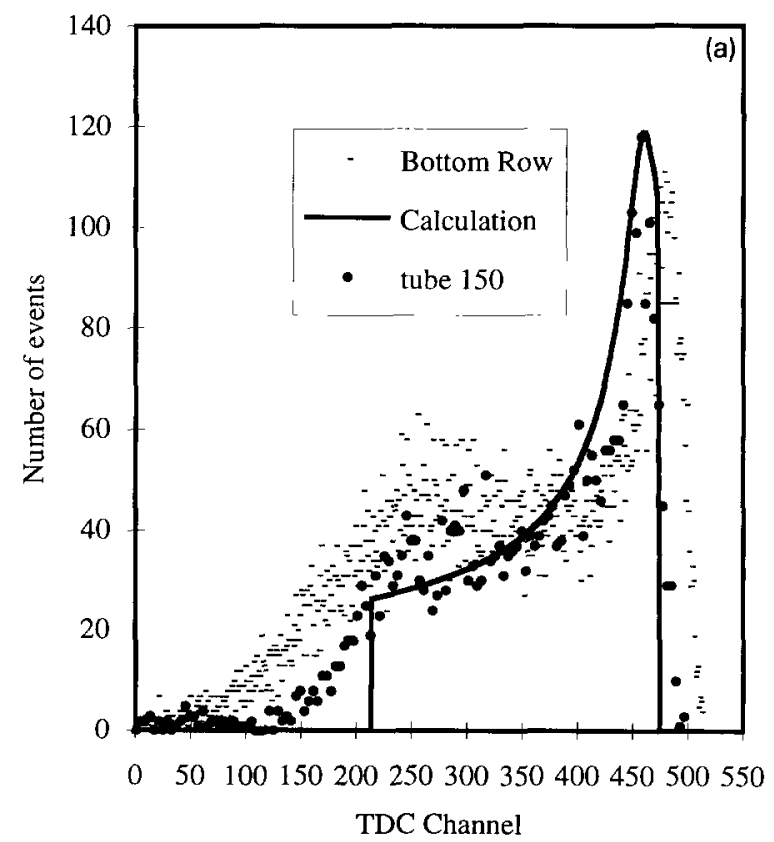

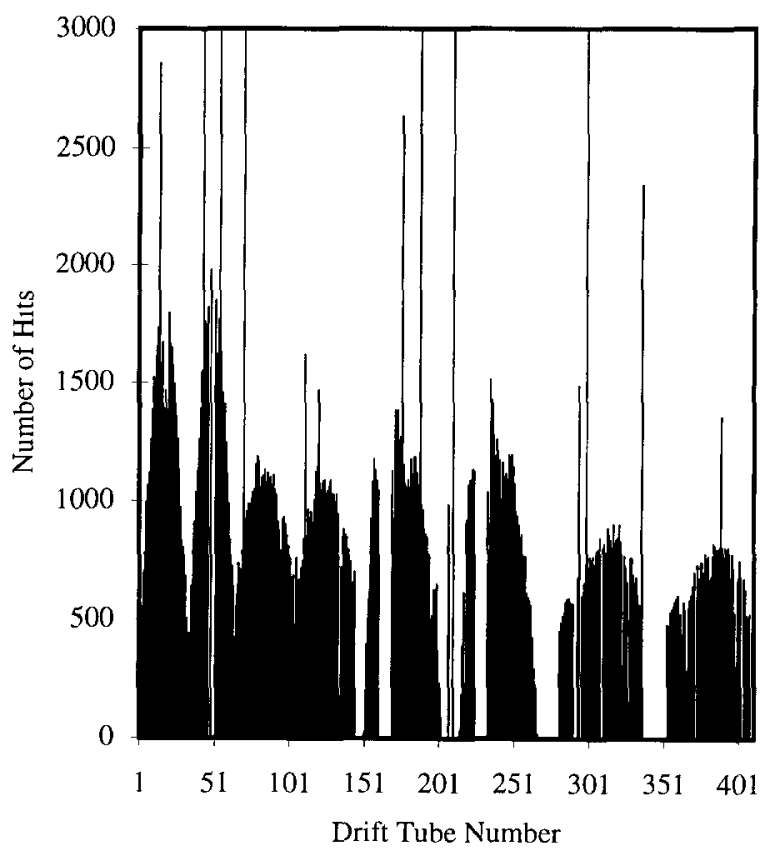

Fig. 11. Drift tubes hit through Pass 13.

imply hits near the wire, and small TDC channels imply hits near the wall. Ideally, there would be a sharp cut-off at a small TDC channel which would

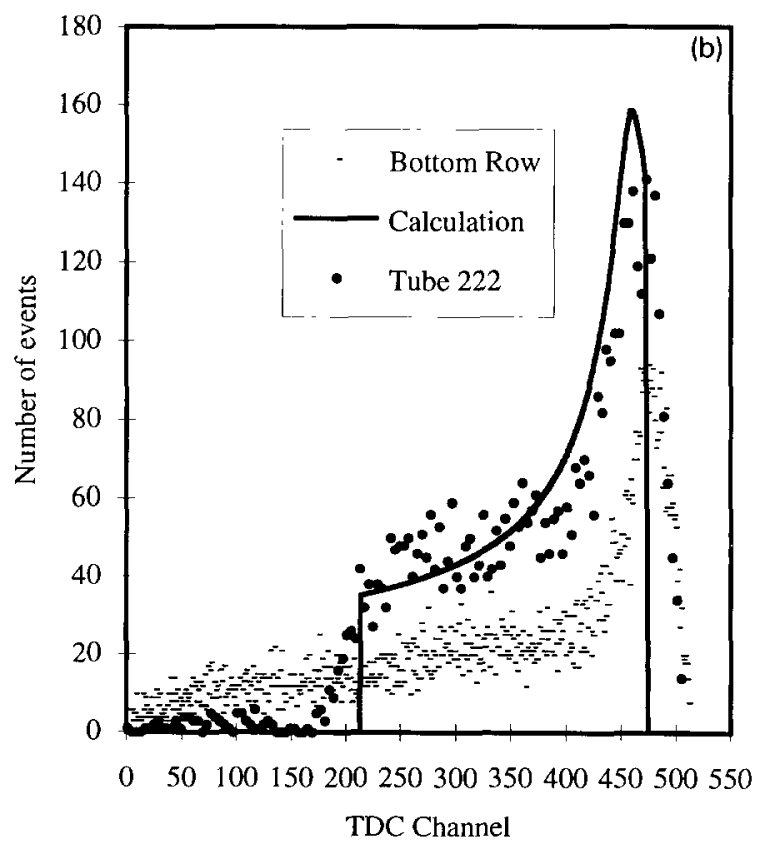

Fig. 12. (a) Drift tube TDC histogram for first half of flight for tube \#150 and for most tubes from the bottom row. Also shown is a calculation of the expected distribution. (b) Same as (a) except for second half of flight (and tube \#222 is used instead of \# 150 ). 
denote the wall, a sharp cutoff at a large TDC channel denoting the wire, and an enhanced density of points near the wire hit channel due to the rapidly increasing electric field near the wire (for a uniform spatial exposure of a drift tube by charged particles, the number of counts per TDC channel is proportional to the drift velocity at the location in the tube corresponding to the TDC channel; the enhanced density of points for large TDC channel corresponds to the increase in drift velocity with electric field - if a saturated gas were used, i.e. one where the drift velocity were independent of field, the TDC vs. drift tube scatter plot would be uniform). In practice, smearing of the TDC channels at the wire and wall will occur due to various sources of time slewing, which in our case is dominated by the large rise time of the scintillator PMT shaping amplifiers (about $100 \mathrm{~ns}$, which corresponds to about 30 TDC channels).

One TDC histogram is shown in Fig. 12a, where the calculated curve of drift velocity as a function of TDC channel is compared to a histogram of data from tubes on the bottom row for the first half of the flight. Also shown are data from the first half of the flight [11] for a particularly nice tube. The features of the data and the calculation are in reasonable agreement. Fig. 12b shows similar plots for the second half of the flight (except tube \#222 is used instead of \#150). Here the agreement of the bottom row data are not as good as for the first half of the flight. There is a noticeable enhancement of small TDC channels, again probably related to electronics noise or after pulsing induced by the larger signals of the iron group nuclei. There also may be a slight enhancement at large TDC channels due to delta ray hits (a heavy cosmic ray nucleus is accompanied by a swarm of delta rays, some of which will always pass close to the wire), but this cannot be too serious a problem since tube \#222 is in excellent agreement with the calculation.

So called "perfect drift tube events" have been used to determine the drift tube resolution. These are events in which there is exactly one hit tube per plane for each of the two projected views. Trajectories were fit as described above, and the root-mean-square of the residuals for the four tubes was calculated. The results for the first half of the flight for the $y$-view (i.e. using the long tubes) are shown in Fig. 13. There is a peak at about $0.6 \mathrm{~mm}$. Half the events have an rms residual of less than $2 \mathrm{~mm}$. We expect that the dominant sources of tracking error are timing walk of the drift tube signals, timing walk of the PMT amplifier signals, alignment errors, multiple scattering, delta ray effects and drift tube amplifier noise which can cause spurious late start triggers at the TDCs. Since the 1-2 mm resolution is adequate for our purposes, no attempt has been made to improve or understand the relative contributions of the various possible sources of error.

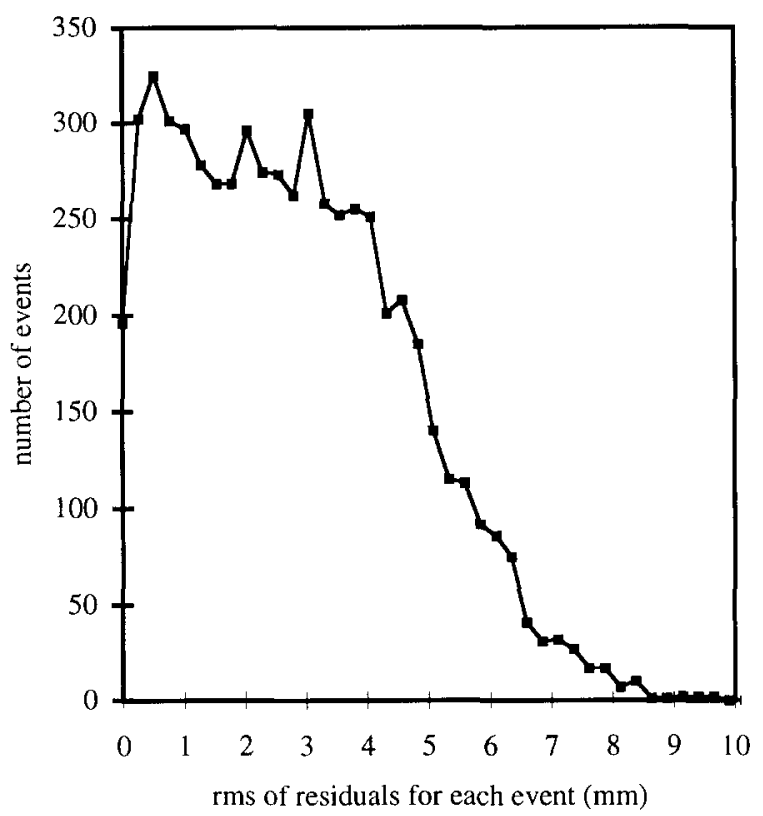

Fig. 13 Root mean square of residuals for perfect drift tube events for long tubes during first half of flight.

\subsection{Scintillator performance}

Using the scintillator maps in Figs. 8 and 9, we are able to calculate a position-corrected geometric mean for each side of each scintillator slab that is hit. For example, if the left side of S1-A is used to determine the corrected scintillation level, this is denoted by s1a1gm and is defined by:

sla1gm

$$
=s 1 \mathrm{a} 1 \times \frac{\exp (\mathrm{S} 1 \mathrm{y} / 58.42 \mathrm{~cm})}{\operatorname{map}_{\mathrm{gm}}(\mathrm{S} 1 \mathrm{x}, \mathrm{S} 1 \mathrm{y}) \times \sqrt{\operatorname{map}_{\mathrm{r}}(\mathrm{S} 1 \mathrm{x}, \mathrm{S} 1 \mathrm{y})}},
$$

where $\operatorname{map}_{\mathrm{gm}}$ is the map value of the geometric mean at the appropriate position, and $\operatorname{map}_{\mathrm{r}}$ is the map value of the ratio of the left-to-right side signals at the appropriate position. This means that each event yields four separate measures of $\mathrm{d} E / \mathrm{d} x$ of the projectile. Except for energy loss fluctuations within a single slab, the four measurements are uncorrelated. Requiring agreement of these four signals is a powerful tool to reject nuclear interactions and events with poorly determined trajectories and map values.

In Fig. 14 we give an example of a comparison between the signals from the two sides of S1-A. Data from Pass 15 for the entire flight have been used. The gaps correspond to the times when high voltages were being changed.

Fig. 15 shows the ratio of the average geometric mean for S1 to that of S2 for Pass 15.2 data. The 


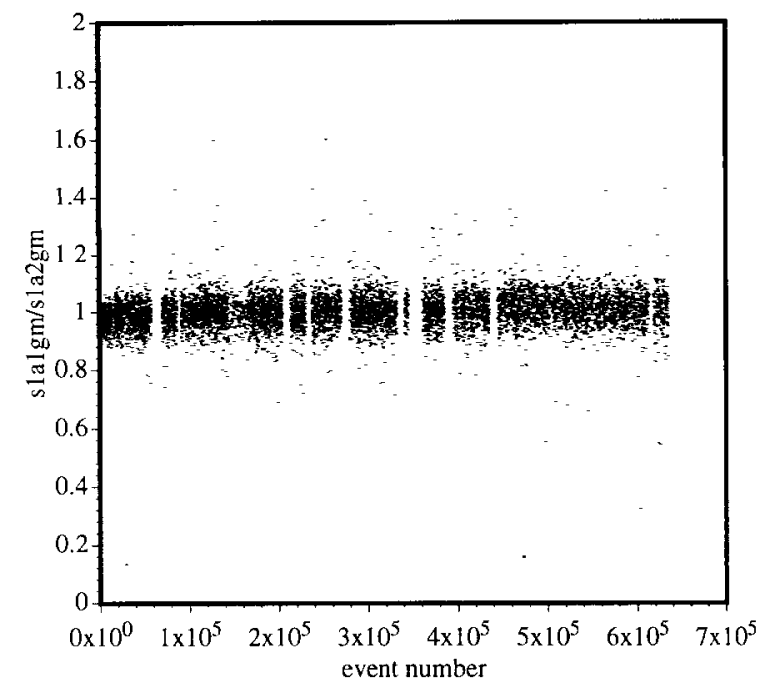

Fig. 14. Ratio of geometric means for the two sides of S1-A calculated using scintillator ratio and geometric mean correction maps in Figs. 8 and 9. Pass 15 data from the whole flight have been used. The gaps correspond to periods when the hıgh voltage was being changed.

average geometric means are calculated by taking the arithmetic average of the geometric means evaluated for the two sides of each slab. The abrupt transitions

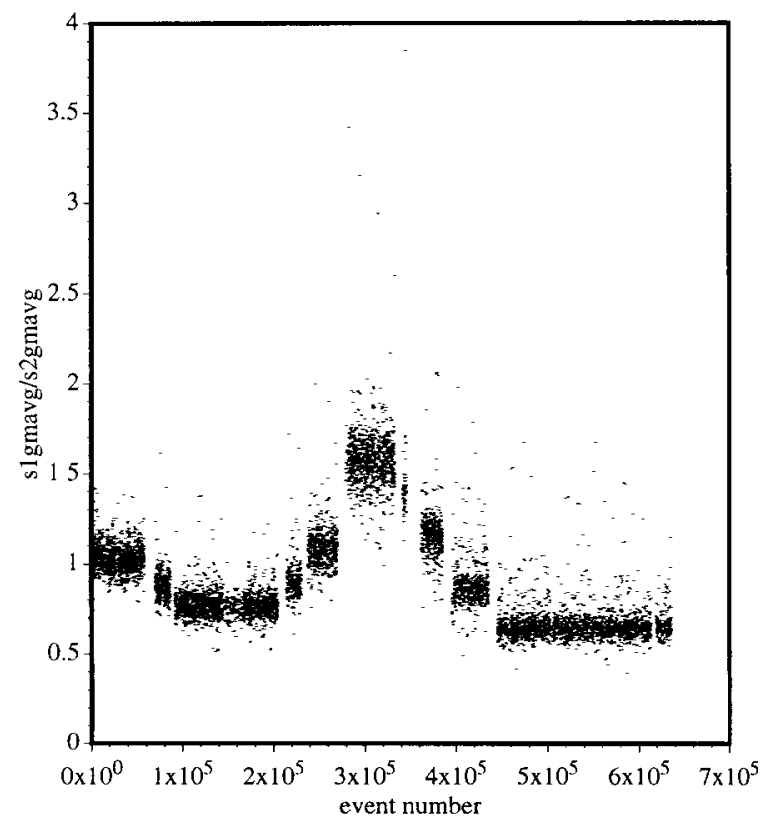

Fig. 15. Ratio of S1 signal to S2 signal for Pass 15.2 data. The time periods $\mathrm{I}$ to $\mathrm{X}$ correspond to the ten stable zones in the figure.

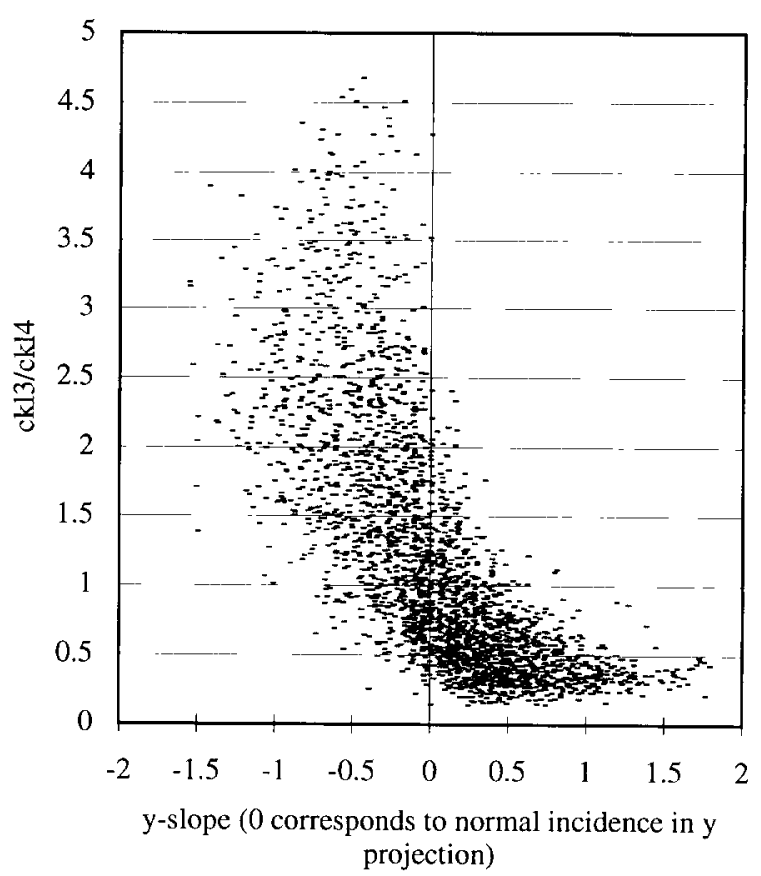

Fig. 16. ckl3/ckl4 as function of the $y$-slope for Pass 21.2 data. The dependence on $x y$-slope is a result of the directionality of Cherenkov radiation. Normal incidence in the $y$ projection corresponds to a $y$-slope of 0 .

are due to high voltage changes. The ten stable zones correspond to the time periods I through $\mathrm{X}$ described in Table 2. Nuclear interactions appear as the scattered points well above the centroids of the stable zones in scatter plot. It is evident that period $\mathrm{X}$ has the largest fraction of iron nuclei, which interact more frequently than lighter cosmic ray particles. The median values for the ten stable zones were used to determine accurately the variation of gain with high voltage. In this way, we were able to correct for high voltage changes which enabled us to combine all data into one set. The normalization convention is that a normally incident fast oxygen nucleus has a geometric mean of 1.00 for each side of each scintillator.

\subsection{Cherenkov counter performance}

To help understand the performance of the Cherenkov counter, we introduce the " $y$-slope", which is the tangent of the projected zenith angle in the plane perpendicular to the long drift tubes. When the $y$-slope is positive, the projectile is moving away from the CKL readout sides ckl1, ckl3, and ckl5. When the $y$-slope is negative the projectile is moving toward ckl1, ckl3, and ck15. Due to the directionality of Cherenkov radiation, one expects that $\mathrm{ckl} 1 / \mathrm{ckl} 2$, ckl3/ckl4, and ckl5/ckl6 will be sensitive to the $y$-slope. That this is 


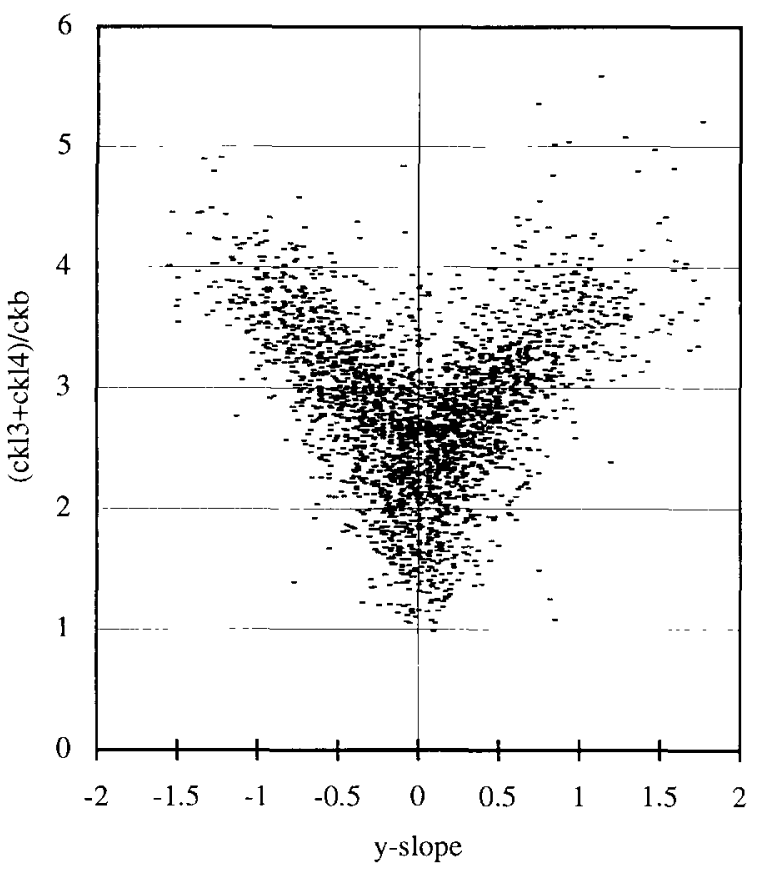

Fig. 17. CKL/CK-LIB ratio as a function of the $y$-slope. The dependence on $y$-slope is a result of the directionality of Cherenkov radiation.

so is shown in Fig. 16, which is a scatter plot of $\mathrm{ckl} 3 / \mathrm{ckl} 4$ for Pass 21.2 data. For normal incidence the ratio varies from 0.5 to 2 . It is apparent that the CKL signals are more sensitive to directionality than to location. This is consistent with the fact that the attenuation length in the Cherenkov radiator is much longer than in the scintillators.

In Fig. 17, we compare the CKL signals with the CK-LIB signal. We have simply taken the sum of the two sides of CKL-B, and we plot the ratio of this to the signal from CK-LIB as a function of the $y$-slope. Units are ADC channels. Again we see a pronounced correlation. When the projectile is normal to $\mathrm{CKL}$, the ratio CKL/CK-LIB varies from 1 to about 3. For large positive or negative $y$-slopes, the ratio is between 3.5 and 4.5. This reflects the fact that for inclined tracks, the Cherenkov light is preferentially collected by the light pipes.

\subsection{Determination of charge with electronic detectors}

To determine charge with the electronic detectors we have adopted the following restrictions and methods of combining S1 and S2, and CKL and CK-LIB signals.

1) We correct all $S 1$ and $S 2$ signals for high voltage changes, normalizing to 830 and $1164 \mathrm{~V}$ respectively, so that fast oxygen at normal incidence has the gm signal of 1.00 for any scintillator side.

2) We require that the left and right side geometric means of each scintillator slab agree with one another within $10 \%$. This eliminates events with badly fit trajectories and in locations of the scintillators with poorly determined mapping parameters.

3) We require that the arithmetic average of the S1 slab geometric means agrees with the arithmetic average of the S2 geometric means within 10\%. This eliminates many, but not all, events with large energy loss fluctuations or nuclear interactions.

4) We define the scintillator signal as:

$S=\frac{2 \text { (S1 leftgm }+ \text { S1 rightgm })+(\text { S2leftgm }+ \text { S2rightgm })}{6}$

if both sides of both scintillator slabs were working properly, and

$S=\frac{4 \text { S1leftgm }+(\text { S2leftgm }+ \text { S2rightgm })}{6}$

for the last half of the flight where one PMT on slab S1-B was malfunctioning. Note that this weighting of $2: 1$ of the S1 and S2 signals is a balance between what would be done to minimize energy loss and photoelectron fluctuations (a $4: 1$ weighting in favor of S1) and what would be done to minimize mapping errors (a $1: 1$ weighting).

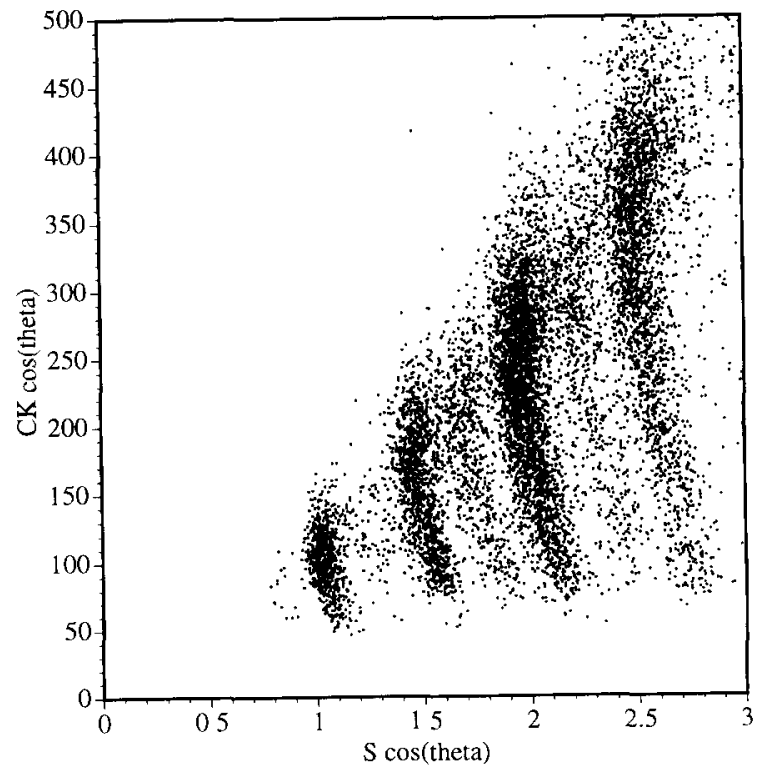

Fig. 18. Cherenkov vs scintillator signal for 10000 events with smallest value of $S \cos \theta$ from Passes 21. The dimensionless Cherenkov signal is defined in the text. The scintillator signal is also defined in the text, and is normalized to unity for minimum ionizing oxygen nucle. 


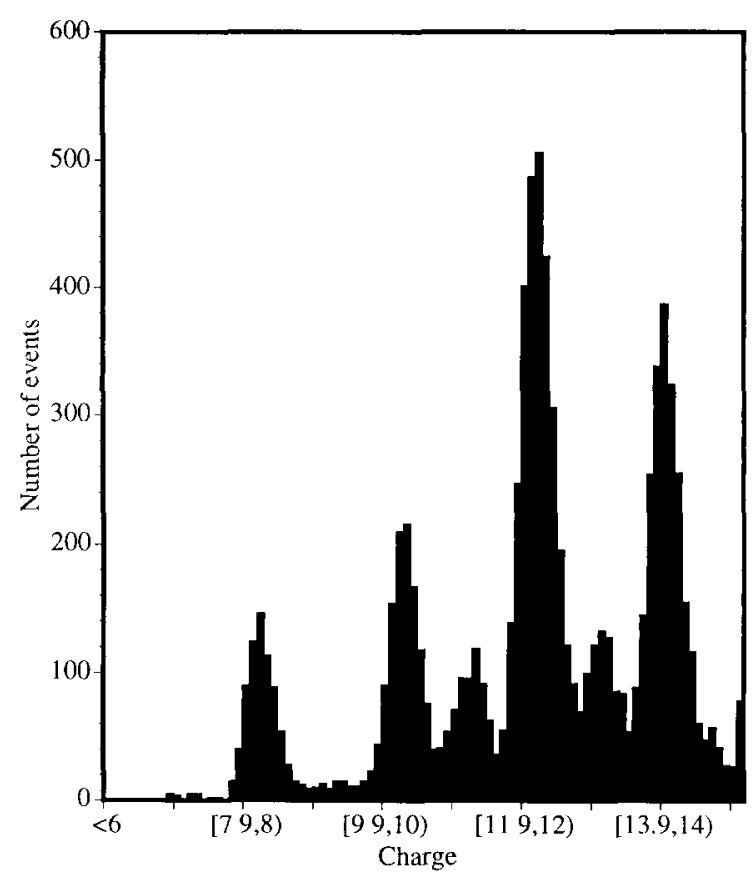

Fig. 19. Charge resolution for first half of flight from the use of the Cherenkov counter and scintillators. No cuts have been made on velocity, position, or zenith angle.

5) We reject events which are below the Cherenkov threshold (i.e. for which the CK-LIB signal is not above the ADC pedestal), since it is expected that low velocity antiparticles will not be able to overcome the galactic wind to penetrate our Galaxy [2].

6) We define the Cherenkov signal as:

$\mathrm{CK}=\frac{\text { CKLIB }+(\text { CKLleft }+ \text { CKLright }) / 2.5}{2}$.

The sum of the CKL PMTs was divided by 2.5 so that the CKLIB and CKL signals would be equal on average for vertical particles (see Fig. 17). No attempt was made to map the Cherenkov response.

7) Both CK and $S$ were multiplied by $\cos \theta$, where $\theta$ is the zenith angle determined by the drift tubes.

A total of 16807 events passed all these cuts.

Fig. 18 shows a $\mathrm{CK} \cos \theta$ vs $S \cos \theta$ scatter plot for the 10000 events with the smallest values of $S \cos \theta$ which survived the above procedure from Passes 21. Fig. 19 shows the corresponding charge histogram. To obtain this, we adopted a slight variation of the BTV model of scintillation [13]. If we denote $Z$ as the magnitude of the charge of the projectile in units of the charge of a proton, $\beta$ as projectile velocity in units of the speed of light, $\gamma$ as the Lorentz factor, $\mathrm{d} E / \mathrm{d} x$ as the stopping power of the projectile in the scintilla- tor, $I=63 \mathrm{eV}$ the mean ionization potential for scintillators to be used in the stopping power formulae, $n=1.52$ the index of refraction of the Cherenkov radiator, $m$ the mass of an electron, $c$ the speed of light, $\epsilon_{\mathrm{m}}=2 m c^{2} \beta^{2} \gamma^{2}$ the maximum energy of a delta ray allowed by kinematics, $T_{0}$ the parameter from the BTV model which characterizes the delta ray energy required to escape the core region of the scintillator around the particle track, $F_{\mathrm{s}}$ the fraction of $\mathrm{d} E / \mathrm{d} x$ which escapes the core region, $B_{\mathrm{s}}$ the parameter from the BTV model which characterizes the degree of radiation induced quenching in the core region, $\mathrm{d} L / \mathrm{d} E$ the dimensionless scintillation efficiency (i.e. the ratio of the total energy of escaping photons to the energy deposited in the scintillator by the projectile), $G$ a scintillator gain factor, $S$ the scintillator signal for normally incident projectiles normalized to 2.5 for silicon, and $\mathrm{CK}$ the Cherenkov signal for a normally incident projectile (in units of channels according to the algorithm described above for combining the light pipe and light box signals), the scintillator and Cherenkov signals are given in our model by:

$\mathrm{CK}=4.6 \mathrm{Z}^{2}\left(1-\frac{1}{\mathrm{n}^{2} \beta^{2}}\right)$,

$S=G \frac{\mathrm{d} E}{\mathrm{~d} x} \frac{\mathrm{d} L}{\mathrm{~d} E}$,

where

$\frac{\mathrm{d} L}{\mathrm{~d} E}=0.03\left(F_{\mathrm{s}}+\frac{1-F_{\mathrm{s}}}{1+B_{\mathrm{s}}\left(1-F_{\mathrm{s}}\right) \frac{\mathrm{d} E}{\mathrm{~d} x}}\right)$,

with

$F_{\mathrm{s}}=\frac{\left(\ln \left(\frac{\epsilon_{\mathrm{m}}}{T_{0}}\right)-\beta^{2}\right)}{2\left(\ln \left(\frac{\epsilon_{\mathrm{m}}}{\gamma I}\right)-\beta^{2}\right)}$

and

$\frac{\mathrm{d} E}{\mathrm{~d} x}=0.165 \frac{\mathrm{MeV}}{\mathrm{g} / \mathrm{cm}^{2}} \frac{\mathrm{Z}^{2}}{\beta^{2}}\left(\ln \left(\frac{\epsilon_{\mathrm{m}}}{I}\right)-\beta^{2}\right) \quad$ if $\gamma<2.5$.

If $\gamma>2.5, \mathrm{~d} E / \mathrm{d} x$ is assumed to have the value according to the above formula for $\gamma=2.5$. Note that all of the relativistic rise in scintillator signal is ac-

Table 4

Parameters used in BTV scintillation model for Bicron-412 scintillator

\begin{tabular}{llll}
\hline Regime & $G\left[\mathrm{~g} / \mathrm{cm}^{2} / \mathrm{MeV}\right]$ & $B_{\mathrm{b}}\left[\mathrm{g} / \mathrm{cm}^{2} / \mathrm{MeV}\right]$ & $T_{0}[\mathrm{keV}]$ \\
\hline $\mathrm{Z} \leqslant 14$ & 0.81 & 0.08 & 30 \\
$\mathrm{Z} \geqslant 14$ & 0.84 & 0.04 & 55 \\
\hline
\end{tabular}




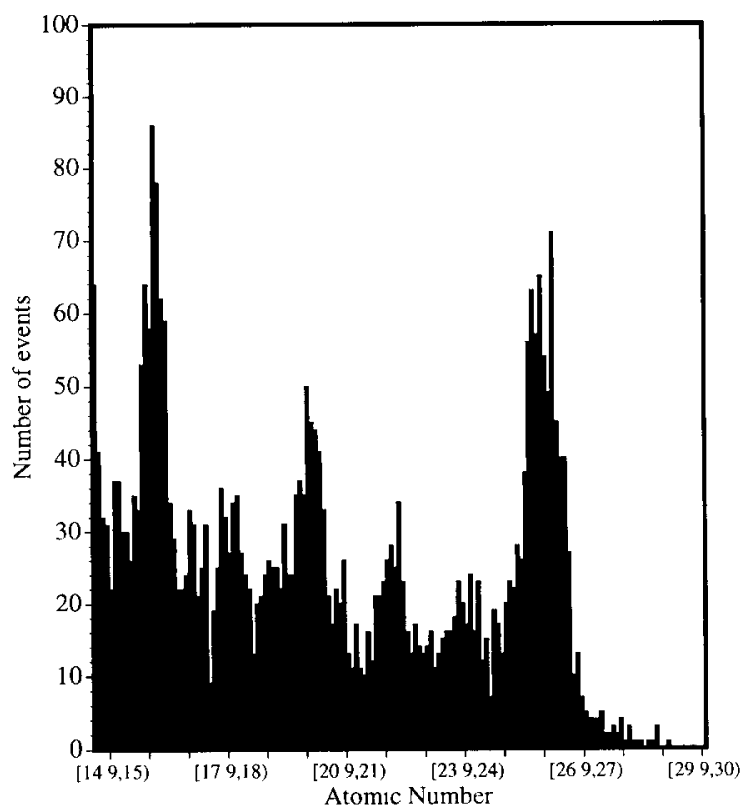

Fig. 20. Histogram for atomic numbers for $Z>14.5$ for entire flight and entire detector. No cuts have been made on energy, angle or detector position (except to exclude events with sub-threshold Cherenkov signals). Charge has been determined from the use of the Cherenkov counter and scintillators.

counted for by the dependence in $F_{\mathrm{s}}$ on Lorentz factor, which has a different form than has been assumed previously [13]. Note also that we have not bothered to include delta ray or scintillation contributions to Cherenkov radiation. These obviously exist, but at a level low enough so as not to be required, especially since Cherenkov resolution is not nearly so critical as scintillator resolution in this experiment.

We were unable to find values of $G, T_{0}$ and $B_{4}$ which fit the data well from oxygen to iron. Therefore we divided the data into two regions: 1$)$ for $Z \leqslant 14$; and 2) for $Z \geqslant 14$. The parameters chosen for the two regimes are given in Table 4 .

Fig. 19 and 20 show the charge histograms resulting from the above model. The charge resolution at silicon is about $0.21 \mathrm{e}$, and that at iron is about $0.38 \mathrm{e}$. The iron resolution is about $0.3 \mathrm{e}$ if we only include data from S1-A, which had both sides working properly during the last half of the flight. The relatively poor resolution one obtains with scintillators for relativistic heavy ions is due to their sensitivity to delta rays and to the suppression of the energy deposited near the core by quenching. Typically charge resolution is about three times worse for $Z>14$ than one would obtain with a detector sensitive to all classes of energy deposition.
Note that no cuts have been made on position, angle, or energy (except to exclude sub-threshold Cherenkov events). The shapes of the charge peaks are characterized by tails on the high side due to energy loss fluctuations, and there is still some residual contamination due to nuclear interactions on the low side. It is important to note that neither of these sources of error in assigning charge with the scintillator data tends to mimic antimatter signatures. Hence the resolution related to the antimatter search is better than it may appear from Figs. 19 and 20.

With the parameters determined above one finds that about $85 \%$ of the energy loss which contributes to scintillation occurs above $T_{0}$. This implies that an anti silicon nucleus would appear to have a scintillator signal at aluminum (about five standard deviations of the scintillator signal), and an anti iron nucleus would appear to have a signal at chromium (also about five standard deviations; see ref. [1] for details). The corresponding Cherenkov signals are the same for matter and antimatter at the same velocity [1].

\section{Analysis of CR-39 detectors}

Following termination of its flight, the experiment came down in heavily wooded and swampy terrain about $150 \mathrm{~km}$ east of Prince Albert, necessitating a helicopter recovery. To prevent the need for the rental of an expensive heavy lift capacity helicopter, which would cause a long delay in recovery, the decision was made to strip the experiment in the forest where it landed to bring its weight down to $1600 \mathrm{~kg}$, which would be light enough to be lifted by a helicopter that was available at the Prince Albert airport. The hemispherical end caps were removed, along with the CAMAC crates and NIM bins, the spreader bar and unnecessary rigging, the gas bottle and regulator, the crush pad and insulation, and everything else that could be removed easily. The experiment was then lifted out by the low lift capacity helicopter. During this process, a tarpaulin which had been attached to the open ends of the gondola midsection was blown off by the strong wind from the helicopter blade, and a large amount of CR-39 from the top array was scattered in the muskeg of the Canadian wilderness. In all, 85 of the 180 top stacks were lost. Fortunately, the top CR-39 is used only as a check on charge at the top of the instrument, in the event that a rare process were to occur in which the charge of a projectile actually increased as it was followed from the top to the bottom of the instrument. This could mimic an antinucleus. Among the possible causes would be an interacting albedo nucleus or a charge exchange interaction which would result in an increased value of charge on the incident projectile. Both of these processes are ex- 
Table 5

Components of Boston microscope system

\author{
Nikon Optıphot 66 inspection microscope with reflectıon and transmission illumination \\ $4 \times, 10 \times, 20 \times, 40 \times, 100 \times$ air objectives \\ New England Affiliated Technologies (NEAT) $15.2 \mathrm{~cm} \times 15.2 \mathrm{~cm}$ travel stage \\ Stepping motor controller (NEAT-202) and joy stick (NEAT-220) for stage \\ Quick-Chek QC1000 digital readout for stage ( $1 \mu \mathrm{m}$ least count) \\ Hitachi KP-M1EK CCD camera $(8.8 \mathrm{~mm} \times 6.6 \mathrm{~mm} ; 756 \times 581$ effective pixels $)$ \\ Hitachi VM- 902 video monitor \\ Scion LG-3 frame grabber \\ Macintosh Quadra 950 with 40 MByte RAM, 400 MByte disk, ethernet (internet) \\ Falcon 2.2 GByte $8 \mathrm{~mm}$ tape drive \\ Apple 21 inch color monitor \\ Apple LaserWriter IIG laser printer \\ "IMAGE" image analysis software available in public domain from NIH \\ Software for Macintosh ("EXCEL", "DeltaGraph", "Softbackup", "NCSA telnet", etc.)
}

pected to be quite rare, and to be very unlikely occurrences for the short flight time. Other than providing this check, the upper CR-39 is useless (if it were used as the primary detector in the antimatter search, numerous antimatter candidates would be identified due to the normal fragmentation of nuclei), and there would be no need to measure any of it. The CR-39 analysis discussed below involves only the bottom CR-39, of which $100 \%$ was recovered.

After the flight, the CR-39 was divided evenly between the University of Michigan and University of California groups. Michigan got the lower three sheets of each stack, and Berkeley got the upper three sheets. These two groups were responsible for etching the plastic, and for scanning and measuring the tracks with fully automated microscope systems that each group had developed. The sheets were etched in $6.25 \mathrm{~N} \mathrm{NaOH}$ for three weeks at $40^{\circ} \mathrm{C}$ in etch tanks with temperature controlled to better than $0.01^{\circ} \mathrm{C}$, and normality to better than $1 \%$. The amount of material removed from the Michigan plastic by general etching $\left(=V_{\mathrm{G}} t\right.$, where $V_{\mathrm{G}}$ is the general etch rate, and $t$ the etch time) was $48.55 \mu \mathrm{m}$ [11]. Following the etch the sheets were rinsed in distilled water, dried and stored in wax paper envelopes. Results of the preliminary work done by these two groups can be found in refs. [9] and [11]. In 1992 (1993) the Michigan (Berkeley) CR-39 was transported to Boston, where measurements are now being carried out with a modest semi-automated microscope system. This system consists of the elements listed in Table 5.

Unless noted otherwise, the CR-39 measurement results discussed below were carried out with the Boston system.

Fig. 21 shows the number of events per CR-39 stack with apparent charge (based on the $S$ vs CK analysis) greater than or equal to 12 . Most of these events should have etched tracks which can be observed and measured with a microscope. Typical event density is $0.4 / \mathrm{cm}^{2}$. The corresponding density of tracks in the CR-39 which are detectable in a scan with a $10 \times$ objective is about $2 / \mathrm{cm}^{2}$. The difference is due to: 1 ) the presence of low energy tracks in the CR-39;2) to heavy nuclei from the first part of the flight which were not recorded in the electronics due to competition with the flux of light particles due to the higher PMT voltages used in the beginning of the flight; and 3) to particles which have the wrong geometry to trigger the electronics. About $2 / 3$ of the CR-39 tracks are easily

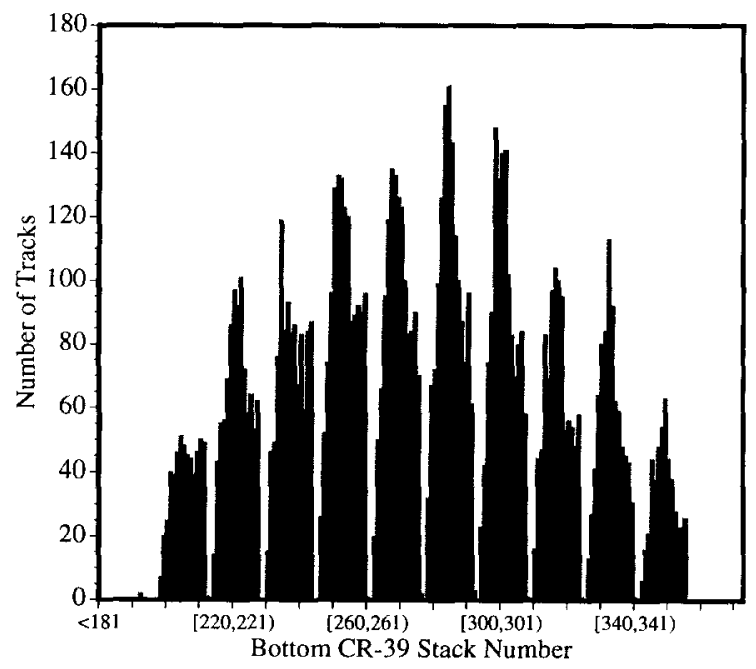

Fig. 21. Numbers of tracks per bottom CR-39 stack for events with the 10000 largest values of $S \cos \theta$. 

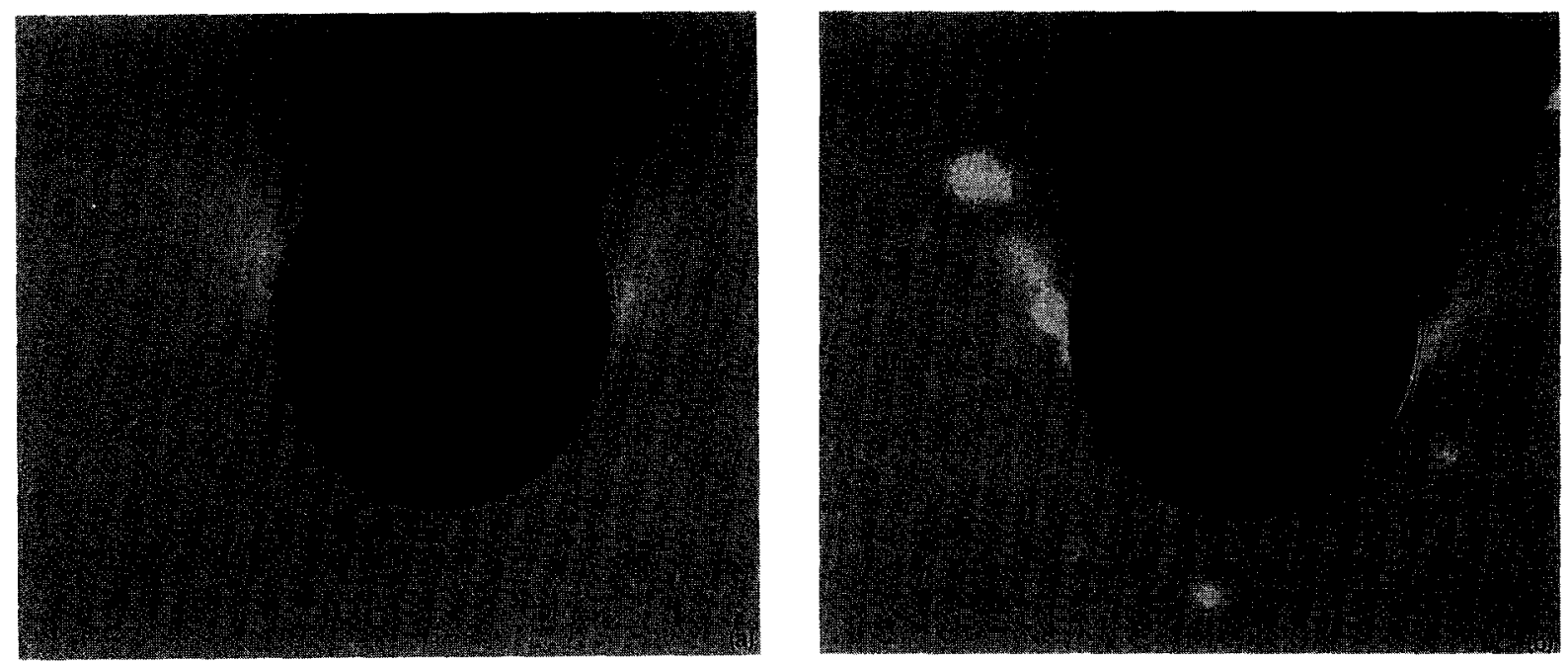

Fig. 22. (a) Image of a track from the top of a top sheet of the bottom CR-39 array (etched in Berkeley). The minor axis is $960 \mu \mathrm{m}$. (b) Image of the cone from the top of the sixth sheet (etched in Michigan) for the same event shown in (a). The minor axis is $101.0 \mu \mathrm{m}$

observed to slow by comparing the cone size at the top of the top sheet with that at the bottom of the bottom sheet (i.e. sixth sheet from the top). In Fig. 22 and 23 we show two typical tracks which do not appear to slow through the six sheets. Fig. 22a is an image of the surface of a large cone, having a minor axis of about $100 \mu \mathrm{m}$, from the top of the top sheet which was processed at Berkeley (fig 22b is for the same particle track at the top surface of the bottom sheet, which was etched at Michigan). Figs, 23a and 23b show images of cones for a less heavily ionizing track (the minor axes being about $45 \mu \mathrm{m}$ ), again for the same top and bottom sheets as for Fig. 22.

The resolution of the CR-39 was demonstrated [9] by comparing cone sizes of the same track on different surfaces, implying a single surface charge standard deviation of $0.30 \mathrm{e}$. This does not include temperature effects, which should be small for EXAM due to the small temperature variations. With the use of cones from all 12 surfaces this implies a potential resolution
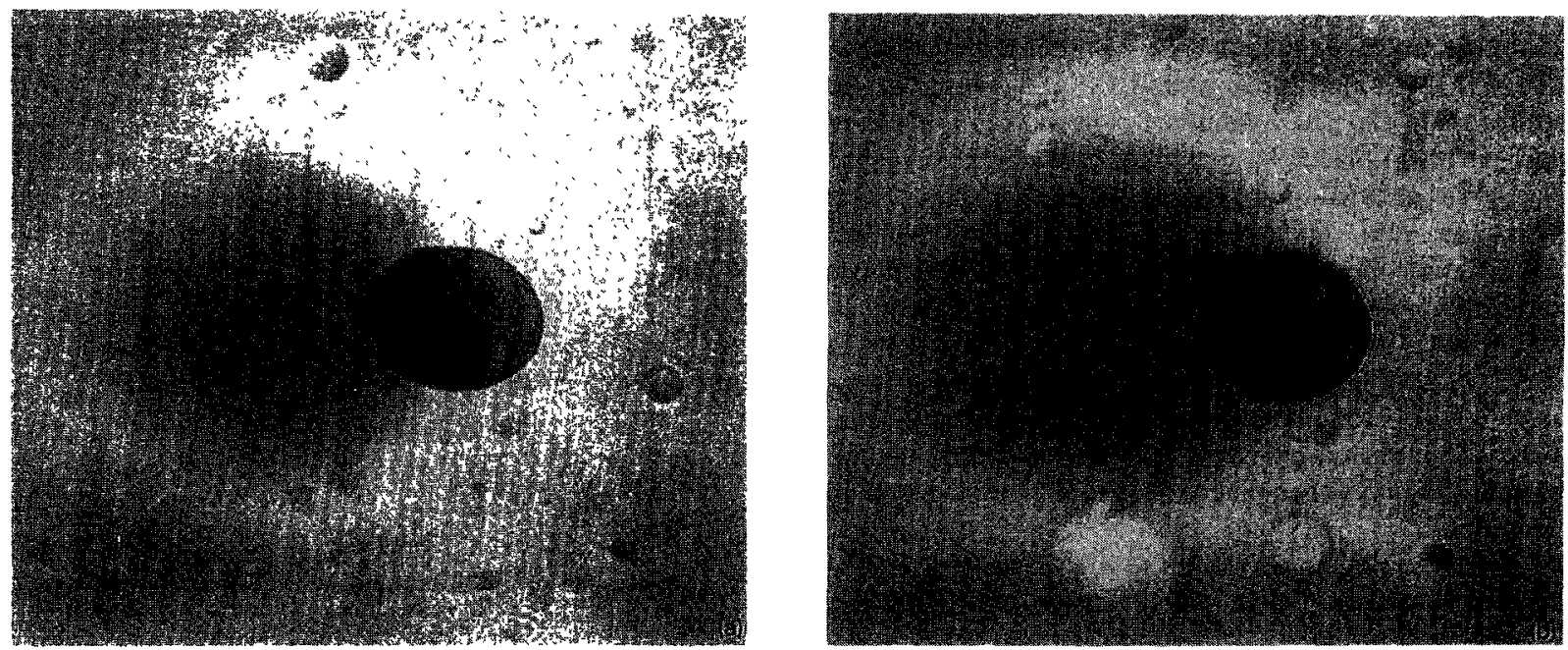

Fig. 23. Same as for Fig. 22, except for a less heavily ionizing partıcle. The minor axes are about $43.5 \mu \mathrm{m}$ and $46.0 \mu \mathrm{m}$ for the (a) Berkeley and (b) Michigan etched sheets, respectively. 
of $0.09 \mathrm{e}$ for the CR-39 when used in conjunction with Cherenkov data.

Since the tracks now being measured in Boston are on sheets that were etched five years ago, an obvious concern is whether the quality of etched cones degrades with time. To put this worry to rest we have recently made measurements of track etch rate of 30 cones from one of the two Bevalac calibration sheets
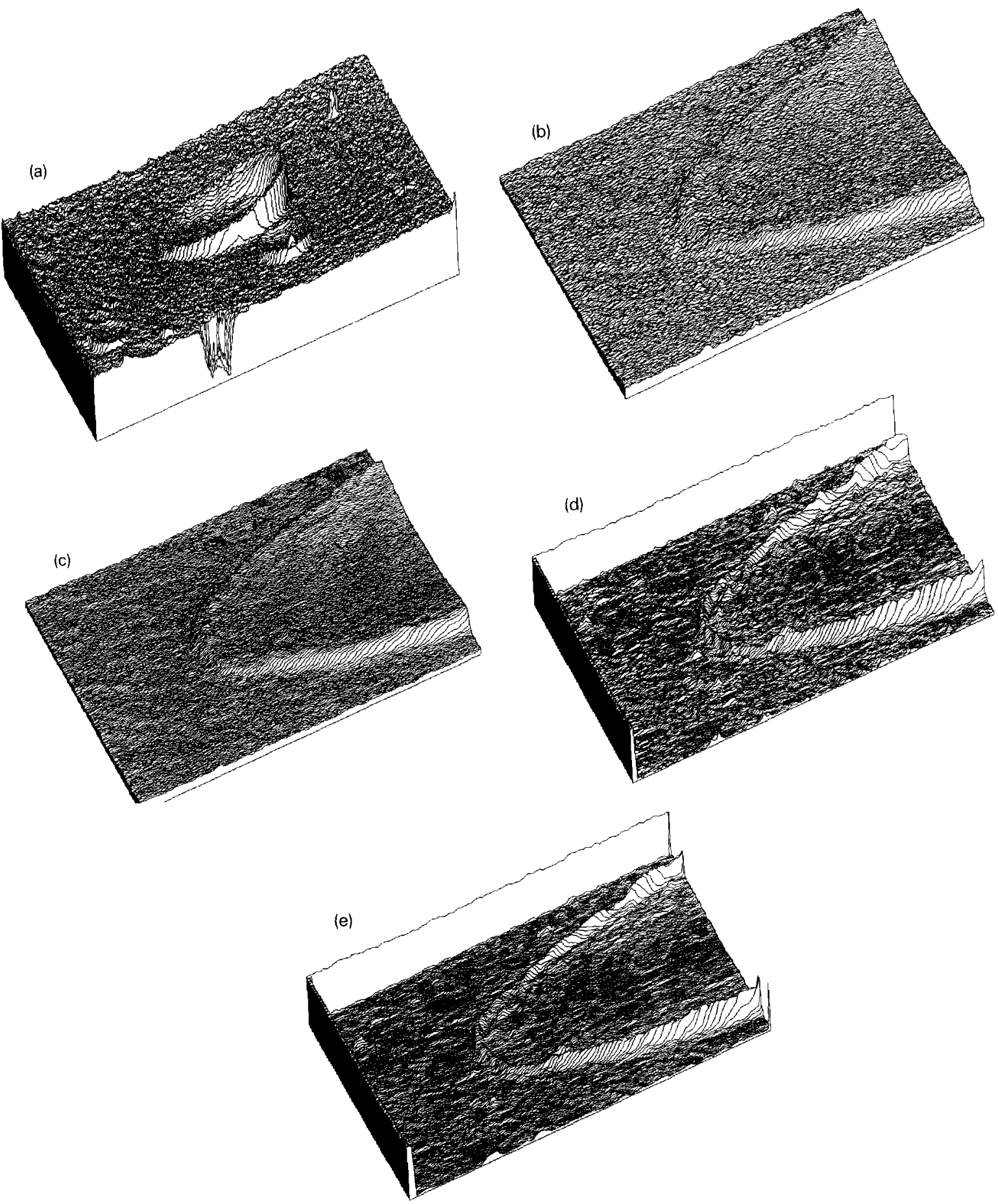

Fig. 24. Images of $1.86 \mathrm{GeV} / \mathrm{amu}$ Ar track for $30^{\circ}$ zenith angle. The minor axıs is $26 \mu \mathrm{m}$. See text for full description. 
used 12 years ago to originally establish the outstanding charge resolution of CR-39 with a relativistic iron beam [4]. The measurements were made of images obtained with a $100 \times$ reflected light air objective in conjunction with the "IMAGE" image analysis program developed by the NIH, and available free of charge in the public domain. We will describe below in more detail the exact algorithms used for the measurements. For now we note that the measurements imply a charge resolution of $0.20 \mathrm{e}$ per surface, compared to the result of $0.24 \mathrm{e}$ per surface determined 12 years ago by measuring minor axes with a filar micrometer.

The Boston microscope system captures images from a CCD camera that are 768 pixels wide $(H)$ by 512 pixels high $(V)$ with 256 gray scale levels. To determine the optimal track measurement procedure we selected a cone from the Bevalac calibration sheet referred to above that is particularly difficult to measure: namely a high inclination track of a lightly ionizing charge, in this case an argon projectile with energy of 1.86 $\mathrm{GeV} / \mathrm{amu}$ and $30^{\circ}$ zenith angle. After trying several different approaches the procedure described below was adopted for making quantitative measurements of track geometry. Figs. 24a-24e show images of this cone that were made with reflected light with a $100 \times$ air objective at various stages in the selected procedure:

Fig 24a shows a surface plot of the raw image. The minor axis is $26 \mu \mathrm{m}$. The height corresponds to decreasing light level (so black is high and white is low).

Fig. 24b shows an expanded view of the surface plot at the shallow side of the track.

Fig. $24 \mathrm{c}$ shows the surface plot after the application of a median filter which reduces noise. With this filter, each pixel is replaced with the median value of its $3 \times 3$ neighborhood.

Fig. 24d shows the surface plot after an edge tracing procedure was applied to the image of Fig. 24c. More precisely, two convolutions are applied to the image, one for a vertical derivative, and one for a horizontal derivative, in which gray scale values of pixels are weighted according to either

$$
\begin{array}{rrr}
1 & 1 & 1 \\
0 & 0 & 0 \\
-1 & -1 & -1
\end{array}
$$

or

$$
\begin{array}{lll}
-1 & 0 & 1 \\
-1 & 0 & 1 \\
-1 & 0 & 1
\end{array}
$$

The convolution giving the larger result is adopted. Fig. 24e shows the surface plot after application of the median filter again.

An ellipse is fit by the IMAGE program to the contiguous set of pixels on the outside of a thresholded image of Fig. 24e.
Once ellipse parameters are obtained, they are corrected for the aspect ratio of 0.946 for the CCD camera/frame grabber system we are using (i.e. a horizontal image of length 100 pixels will be measured to have a length of 94.6 pixels if the CCD camera is rotated by $90^{\circ}$ ). The length conversion factor for the corrected pixel measure (where the horizontal scale is regarded as fixed) is 5.30 pixels per $\mu \mathrm{m}$ for our $100 \times$ air objective.

Once the corrected ellipse parameters are obtained, relevant cone parameters can be easily obtained with the following formulae, where $s=V_{\mathrm{t}} / V_{\mathrm{G}}$ (the ratio of track etch rate to general etch rate), $\theta$ is the zenith angle, $b=$ the cone minor axis, $a=$ cone major axis, $B=b /\left(2 V_{\mathrm{G}} t\right), A=a /\left(2 V_{\mathrm{G}} \mathrm{t}\right)$, and $\Delta y$ is the distance between the steep edge of the cone and the point at which the particle trajectory cut the post-etch surface (measured along the major axis):

$\cos \theta=\frac{1+B^{2}}{\left[\left(1-B^{2}\right)^{2}+4 A^{2}\right]^{1 / 2}}$

and

$s=\frac{\left[\left(1-B^{2}\right)^{2}+4 A^{2}\right]^{1 / 2}}{1-B^{2}}$

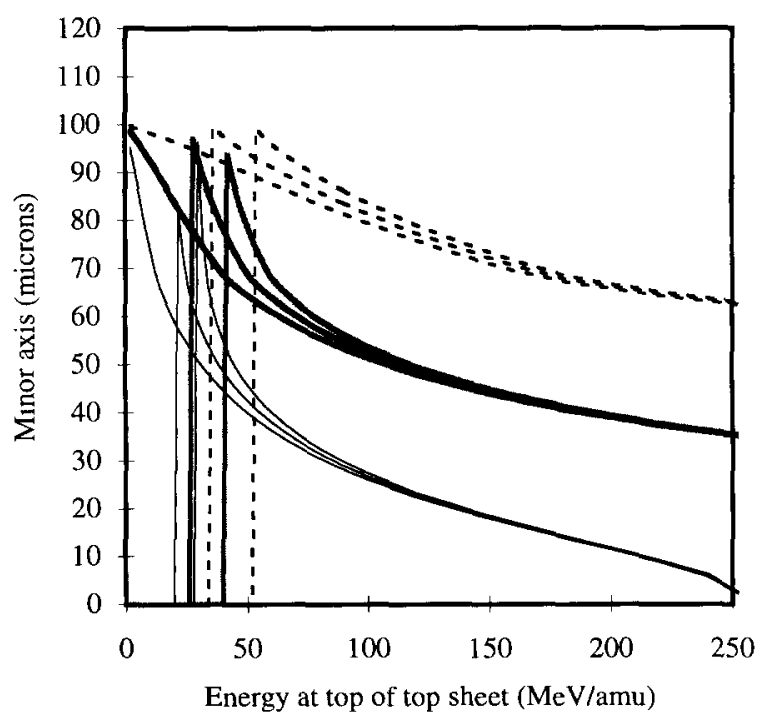

Fig. 25. Calculated response of CR-39 for zenith angle $=30^{\circ}$ for iron (dashes), silicon (shaded), oxygen (solid) for top of top sheet, bottom of third sheet, and bottom of bottom sheet in a six sheet stack. Relativistic iron is assumed to have a minor axis of $42 \mu \mathrm{m}$. 
and

$\Delta y=V_{\mathrm{G}} t \frac{1-B^{2}}{1+B^{2}} \sqrt{A^{2}-B^{2}}$.

The inverse relationships are:

$b=2 V_{\mathrm{G}} t \sqrt{\frac{s \cos \theta-1}{s \cos \theta+1}}$

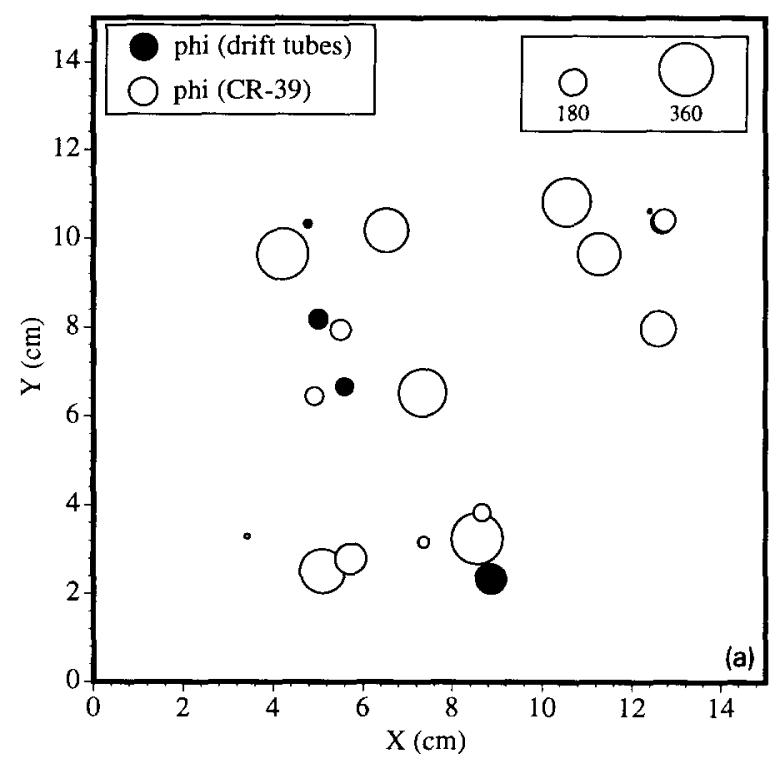

and

$a=2 V_{\mathrm{G}} t \frac{\sqrt{s^{2}-1}}{s \cos \theta+1}$

and

$\Delta y=\frac{V_{\mathrm{G}} t \sin \theta}{\cos \theta(s \cos \theta+1)}$.

We have determined resolution for zenith angle (using the formula above for $\theta$ ) and azimuth angle

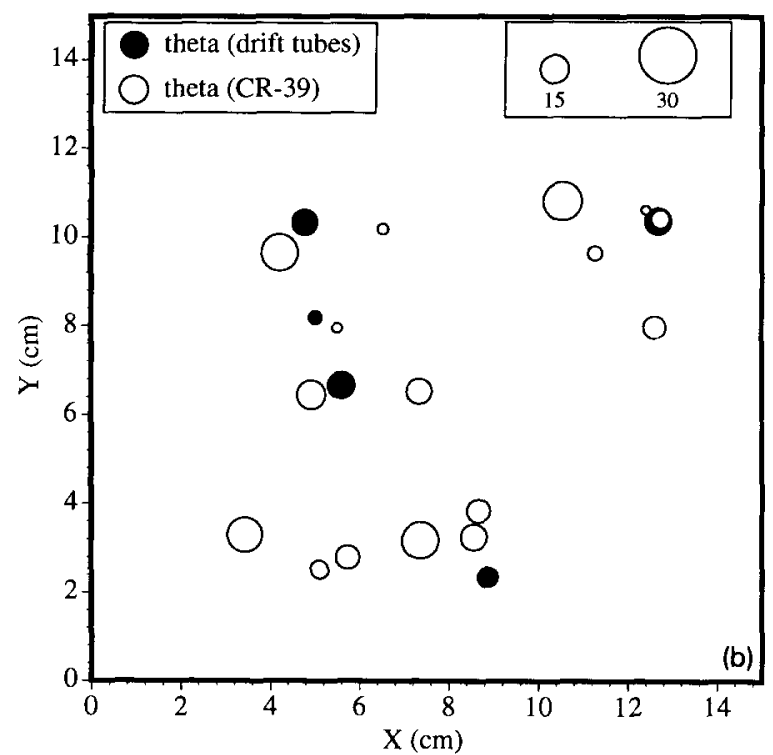

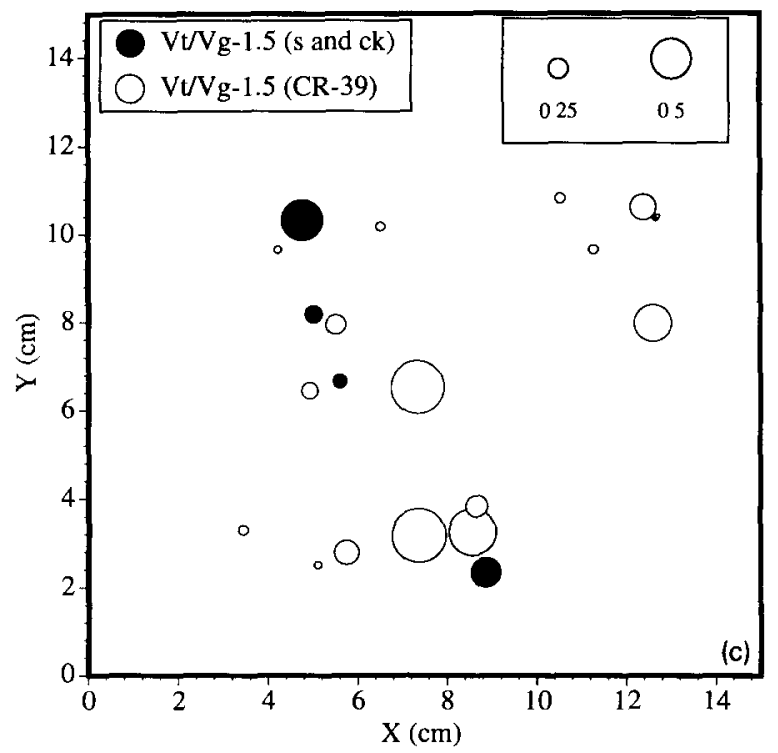

Fig. 26. (a) Comparison of azimuth angle for drıft tube and CR-39 candidates in stack 284. (b) Comparison of zenith angle for drift tube and CR-39 candidates in stack \#284. (c) Comparison of $V_{\mathrm{t}} / V_{\mathrm{G}}-1.5$ for drift tube and CR-39 candidates in stack \#284. 
(returned by the IMAGE ellipse fitting routine and corrected for non unity aspect ratio) by measuring 100 tracks from the original Bevalac calibration sheet. For each angle, we found that accuracies of the order of a few tenths of a degree are possible. This accuracy degrades to a few degrees for zenith angles of the order of $15^{\circ}$ or less.

Knowledge of zenith and azimuth angles from CR-39 cones is important in establishing which CR-39 tracks are associated with which drift tube tracks. Another handle on matching events is a comparison of $V_{t} / V_{G}$ with the scintillation signal. This point is illustrated with Fig. 25, which shows the calculated minor axes of cones for oxygen, silicon, and iron nuclei at $30^{\circ}$ at various energies, and at various positions in a stack of six sheets of CR-39 with the same thickness as used for the flight. The tracks have been normalized to $42 \mu \mathrm{m}$ as the value of the minor axis of a relativistic iron at $30^{\circ}$ (a relativistic manganese nucleus at $30^{\circ}$ would have a minor axis of $40.5 \mu \mathrm{m}$ ). The response function assumed is from a batch of CR-39 different from that flown, and may be different from the one for the EXAM plastic, but our conclusions here are independent of such details. If we suppose that it is known from electronic data that an event in question has a charge within one unit of iron, that it has a zenith angle of $30^{\circ}$, and that it has a large velocity, we see that there is an energy range of only a few $\mathrm{MeV} / \mathrm{amu}$ for silicon that would be consistent with such a prediction, and that no oxygen event could simulate such a fast iron (because it would demonstrate considerable slowing throughout the six sheet stack). So accidental backgrounds from the abundant silicon and oxygen species will not pose a problem if etch rate is taken into account.

\section{Matching events in CR-39 with those in drift tubes}

We have used stack \#284 to establish our ability to match tracks in the CR-39 with those in the electronic detectors. We restricted our attention to highly charged tracks with $\theta<15^{\circ}$, and to the central $10.2 \mathrm{~cm} \times 10.2$ $\mathrm{cm}$ section of the sheet. There were five drift tube events heavier than argon which unambiguously satisfied these conditions (candidates with drift tube tracks which could conceivably have hit the CR-39 outside of the area scanned were not considered). Scanning of the CR-39 was done manually with a Nikon SC microscope, with a $4 \times$ objective and $10 \times$ eyepieces and having a large depth of field. The six CR-39 sheets were superimposed, enabling true events to be readily identified (the 12 cones literally "leap out" at the scanner). Tracks were only considered which were not obviously slowing, and which had minor and major axes agree within $4 \%$. The scanning process took several hours. Sixteen CR-39 candidates were found. Their cones on the top of the top Michigan sheet were measured with the procedure described above with the $100 \times$ M plan air objective. Figs. 26a-c show bubble chart comparisons of azimuth angle, zenith angle, and predicted $\left(V_{\mathrm{t}} / V_{\mathrm{G}}-1.5\right)$ for the CR-39 and electronic events, as functions of position determined either with CR-39 or the drift tubes. Bubble diameters are proportional to the various quantities. To evaluate track etch rate for the CR-39 measurements we assumed that $V_{\mathrm{G}} t=48.55 \mu \mathrm{m}$ [11]. To evaluate track etch rate from the electronic events we assumed that

$\frac{V_{\mathrm{t}}}{V_{\mathrm{G}}}=1.60\left(\frac{Z / \beta}{26}\right)^{067}$,

which is similar (but not identical) to the response of the original Bevalac calibration [4]. We also used the scintillation and Cherenkov model described in detail above to determine $Z$ and $\beta$.

Perusal of Fig. 26 shows without much effort that four matches are easily identified. The fifth drift tube event also appears to be identified with a CR-39 event, except that the CR-39 cone is much too small, indicating possibly that a nuclear interaction occurred. Details of the matches are given in Tables 6 and 7.

The drift tube tracks for the four matched events are shown in Figs. 27a and 27b. The most suspicious fit out of the eight shown is the $x$ view for event 501326 . This is because it has two small TDC channels (corresponding to apparently large impact parameters) near the CR-39. As we have seen before, iron type signals have some problems with after pulsing which tend to make impact parameters appear larger than they should be. Thus one could just as easily have the correct $x$ value at the CR-39 shift downward by several cm (but

Table 6

Geometric details of matched events for the middle part of stack \# 284 (d $t$ means drift tube)

\begin{tabular}{lcccccccc}
\hline Event & $\begin{array}{l}x(\mathrm{~d} t) \\
{[\mathrm{cm}]}\end{array}$ & $\begin{array}{l}x(\mathrm{CR}-39) \\
{[\mathrm{cm}]}\end{array}$ & $\begin{array}{l}y(\mathrm{~d} t) \\
{[\mathrm{cm}]}\end{array}$ & $\begin{array}{l}y(\mathrm{CR}-39) \\
{[\mathrm{cm}]}\end{array}$ & $\begin{array}{l}\varphi(\mathrm{d} t) \\
{[\mathrm{deg}]}\end{array}$ & $\begin{array}{l}\varphi(\mathrm{CR}-39) \\
{[\mathrm{deg}]}\end{array}$ & $\begin{array}{l}\theta(\mathrm{d} t) \\
{[\mathrm{deg}]}\end{array}$ & $\begin{array}{l}\theta(\mathrm{CR}-39) \\
{[\mathrm{deg}]}\end{array}$ \\
\hline 501326 & 8.86 & 5.74 & 2.34 & 2.79 & 211 & 205 & 11 & 13 \\
515849 & 5.59 & 4.93 & 6.65 & 6.45 & 125 & 121 & 15 & 15 \\
621736 & 5.00 & 5.51 & 8.18 & 7.95 & 137 & 134 & 8 & 5 \\
575339 & 12.67 & 12.73 & 10.36 & 10.41 & 158 & 148 & 15 & 10 \\
\hline
\end{tabular}


Table 7

Detector signal detals for matched events in stack \#284 (the last column is the ratio of $(Z / \beta)$ as determined by the $\mathrm{Cr}-39$ to that determined by the scintillators and Cerenkov radiator

\begin{tabular}{lllllll}
\hline Event & $\begin{array}{l}S \\
\times \cos \theta\end{array}$ & $\begin{array}{l}V_{\mathrm{t}} / V_{\mathrm{g}} \\
(\mathrm{CR}-39)\end{array}$ & $\begin{array}{l}\mathrm{CK} \\
\times \cos \theta\end{array}$ & $\begin{array}{l}Z / \beta \\
\mathrm{S} \text { and } \\
\mathrm{CK}\end{array}$ & $\begin{array}{l}Z / \beta \\
\mathrm{CR}-39\end{array}$ & $\begin{array}{l}\mathrm{CR}-39 / \\
\text { scint }\end{array}$ \\
\hline 501326 & 7.19 & 1.807 & 888 & 32.98 & 31.22 & 0.95 \\
515849 & 6.34 & 1.709 & 1136 & 28.05 & 28.70 & 1.02 \\
621736 & 6.21 & 1.748 & 941 & 2925 & 29.70 & 1.02 \\
575339 & 5.24 & 1.550 & 883 & 25.54 & 24.79 & 0.97 \\
\hline
\end{tabular}

not upward!). This is consistent with the bad match of $x$ coordinates for this event as seen in Table 6 .

\section{Conclusions}

There are about 10000 events that we have electronic data for, and that should have tracks in the CR-39 sheets. During the next year we will scan and measure the bottom array of CR-39 to identify matches, and to determine the resolution of the CR-39/CK combination in measuring charge. We have presented results in this paper which are very encouraging that the resolution will be good enough to enable us to complete our search for antimatter at a sensitivity of about one part in 10000 .

\section{Acknowledgements}

This experiment was supported by several grants from the Particle Physics Division of the National Science Foundation, and by balloon operations support from the National Aeronautics and Space Administration. We thank Michael Solarz, Ruth Claxton, Ric Bruni, Fred Wittel, Elida Obaldia, Jan Reynoldson, Jeff Wilkerson, George Patterson, Todd Karakashian, Doug Lowder and Steve Barwick for valuable contributions. We thank George Brandenberg for permitting us to use the Harvard University High Energy Physics Laboratory to prepare EXAM for flight. And we thank Danny Ball, Johnny Mack, and the others of the National Scientific Balloon Facility's launch crew for their tenacity in getting off the EXAM flight in a particularly difficult launch campaign.

\section{References}

[1] S.P. Ahlen, P.B. Price, M.H. Salamon and G. Tarlé, Nucl. Instr. and Meth. 197 (1982) 485.
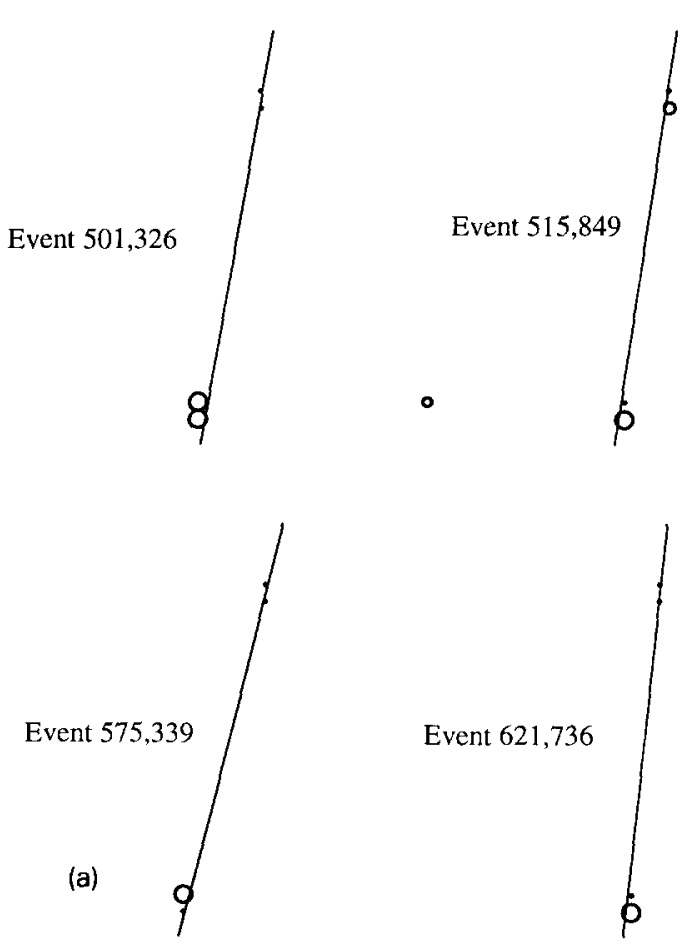
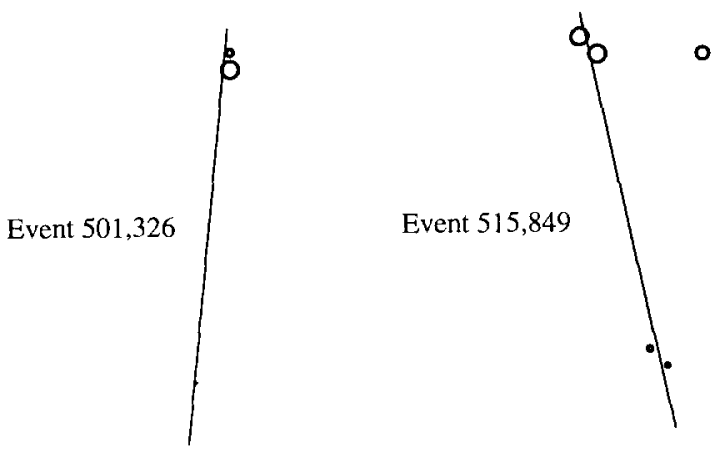

Fig. 27. (a) $x$-view of drift tube fits for the matched events of stack 284. (b) $y$-view of drift tube fits for the matched events of stack \#284. In the figures, $x$ and $y$ increase as one moves to the right. 
[2] S.P. Ahlen, P.B. Price, M.H. Salamon and G. Tarlé, Astrophys. J. 260 (1982) 20.

[3] N. Lund and M. Rotenberg, Proc. 19th Int. Cosmic Ray Conf., LaJolla, CA, USA, NASA, eds. F.C. Jones, J, Adams and G.M. Mason, Conf. Publ. no. 2376, vol. 2 (1985) p. 330.

[4] G. Tarlé, S.P. Ahlen and P.B. Price, Nature 293 (1981) 556.

[5] S.P. Ahlen, B.G. Cartwright and G. Tarlé, Nucl. Instr. and Meth. 143 (1977) 513.

[6] S.P. Ahlen and T.E. Coan, US Patent \#5 151963 (1992).

[7] Y. Asano et al., Nucl. Instr. and Meth. A 254 (1987) 35.

[8] A. Tomasch, S.P. Ahlen, C. Bower, P.B. Price, M.H.
Salamon, G. Tarlé, J. Musser and H. Crawford, Nucl. Instr. and Meth. A 241 (1985) 265.

[9] D.P. Snowden-Ifft et al., Proc. 21st Int. Cosmic Ray Conf., Adelaide, Australia, ed. R.J. Protheroe, vol. 3 (1990) p. 304.

[10] T.E. Coan, Ph.D. Thesis, Univ. of California at Berkeley, Berkeley, CA, USA (1989).

[11] D.J. Crary, Ph.D. Thesis, University of Michigan, Ann Arbor, MI, USA (1991).

[12] I. Lehraus, R. Matthewson and W. Tejessy, Nucl. Instr. and Meth. 200 (1982) 199.

[13] M.H. Salamon and S.P. Ahlen, Nucl. Instr. and Meth. 195 (1982) 557. 